\title{
Impulsivity and comorbid traits: a multi-step approach for finding putative responsible microRNAs in the amygdala
}

\author{
Andrzej Z. Pietrzykowski ${ }^{1,2 *}$ and Sabine Spijker ${ }^{3 *}$ \\ ' Department of Animal Sciences, Rutgers University, New Brunswick, NJ, USA \\ ${ }^{2}$ Department of Genetics, Rutgers University, Piscataway, NJ, USA \\ ${ }^{3}$ Department of Molecular and Cellular Neurobiology, Center for Neurogenomics and Cognitive Research, Neuroscience Campus Amsterdam, VU University, \\ Amsterdam, Netherlands
}

\author{
Edited by: \\ Robert W. Williams, University of \\ Tennessee Health Science Center, \\ USA \\ Reviewed by: \\ Camron D. Bryant, Boston \\ University School of Medicine, USA \\ Jun Xu, Washington State \\ University, USA \\ *Correspondence: \\ Andrzej Z. Pietrzykowski, \\ Department of Animal Sciences, \\ Rutgers University, 67 Poultry Farm \\ Lane, New Brunswick, NJ 08901 , \\ USA \\ e-mail: andrepi@aesop.rutgers.edu; \\ Sabine Spijker, Department of \\ Molecular and Cellular Neurobiology, \\ Center for Neurogenomics and \\ Cognitive Research, Neuroscience \\ Campus Amsterdam, VU University, \\ De Boelelaan 1085, 1081 HV \\ Amsterdam, Netherlands \\ e-mail:s.spijker@vu.nl
}

Malfunction of synaptic plasticity in different brain regions, including the amygdala plays a role in impulse control deficits that are characteristics of several psychiatric disorders, such as ADHD, schizophrenia, depression and addiction. Previously, we discovered a locus for impulsivity (Impu1) containing the neuregulin 3 (Nrg3) gene, of which the level of expression determines levels of inhibitory control. MicroRNAs (miRNAs) are potent regulators of gene expression, and have recently emerged as important factors contributing to the development of psychiatric disorders. However, their role in impulsivity, as well as control of Nrg3 expression or malfunction of the amygdala, is not well established. Here, we used the GeneNetwork database of BXD mice to search for correlated traits with impulsivity using an overrepresentation analysis to filter for biologically meaningful traits. We determined that inhibitory control was significantly correlated with expression of miR-190b, -28a, -340, -219a, and -491 in the amygdala, and that the overrepresented correlated traits showed a specific pattern of coregulation with these miRNAs. A bioinformatics analysis identified that miR-190b, by targeting an Nrg3-related network, could affect synaptic plasticity in the amygdala, targeting bot impulsive and compulsive traits. Moreover, miR-28a, $-340,-219 a$, and possibly -491 could act on synaptic function by determining the balance between neuronal outgrowth and differentiation. We propose that these miRNAs are attractive candidates of regulation of amygdala synaptic plasticity, possibly during development but also in maintaining the impulsive phenotype. These results can help us to better understand mechanisms of synaptic dysregulation in psychiatric disorders.

Keywords: impulsive action, miR-28a, miR-340, miR-219a, miR-491, miR-346, recombinant inbred strain, bioinformatics

\section{INTRODUCTION}

Impulsivity is a personality trait that often occurs in psychiatric disorders. It is one of the hallmarks of attention deficit hyperactivity disorder (ADHD) (Barkley, 1997), substance abuse, bipolar disorder and schizophrenia (McElroy et al., 1996; Koob and Le Moal, 1997; Nestor, 2002; Koob and Volkow, 2010; Lombardo et al., 2012). Increase in impulsivity has been also reported in brain injuries (Bechara and Van der Linden, 2005), in Parkinson's disease secondary to dopaminergic therapy (Wolters et al., 2008; Callesen et al., 2013), or Alzheimer's disease (Rochat et al., 2008). Impulsivity, or the lack of impulse control, is a multifactorial construct (Evenden, 1999) that involves both impulsive choice, and impulsive action; the latter also referred to as the absence of inhibitory control. High impulsive choice is measured by an increased preference for an immediate small over a larger but delayed reward that is more beneficial for the individual. On the other hand, inhibitory control is the ability to inhibit or hold back a prepotent response. Thus, a high level of impulsive action is characterized by poorly conceived, prematurely expressed, unduly risky, or inappropriate actions that often result in undesirable consequences in the long-term. Emotional responses typical for impulsive behavior are orchestrated by several neuronal structures, among which the amygdala is in part responsible for impulsive choice (Gupta et al., 2011) and impulsive action (Depue et al., 2014), as well as for the compulsive component, like addiction (Koob, 2009; Koob and Volkow, 2010). The comorbid nature of impulsivity reflects a multifactorial etiology of psychiatric disorders, and knowledge of its full biological underpinning remains scarce.

Current models explaining psychiatric illnesses have abandoned simple neurotransmitter models, and rather hold disruptions across whole cellular networks responsible. In this view, amongst other factors contributing, microRNAs (miRNAs), which could modulate expression of network of genes and proteins, have the potential to bridge the current gap in our knowledge between the treatment of these debilitating disorders and the underlying neurobiology. After the discovery of the first miRNA in the early nineties (Lee et al., 1993; Wightman et al., 1993), these 18-25 nucleotides long, single-stranded, noncoding RNA species, have emerged as important regulators of 
post-transcriptional gene expression (Ambros and Lee, 2004; Landgraf et al., 2007; Friedman et al., 2009), including constituents of synaptic structures within neuronal circuits (Siegel et al., 2011). Typically, a miRNA can target multiple mRNAs, by binding to its specific miRNA binding site located within $3^{\prime}$-untranslated region ( $3^{\prime}$-UTR) of each targeted mRNA (Farh et al., 2005; Lewis et al., 2005). This way a single miRNA can regulate activity of multiple members of a complex biological network. Although miRNAs have been linked recently to the development of psychiatric disorders (Im and Kenny, 2012; Xu et al., 2012; Nestler, 2014), neurodegenerative diseases (Hébert and De Strooper, 2009), and actions of drugs of abuse and alcohol (Pietrzykowski, 2010), a role for miRNAs in impulsivity is not well established. Thus, far, only some indirect evidence suggests that miRNAs may play a role in this trait. An association between two single nucleotide polymorphisms (SNPs) located within the miR96 gene and attention deficit/hyperactivity disorder (ADHD) was reported (Sánchez-Mora et al., 2013). Additionally, an association between trait impulsivity using self-reporting measures and SNPs located in a genomic region encoding the $3^{\prime}$-UTR of the SNAP25 mRNAs that contain the miR-641 binding site was described (Németh et al., 2013).

Recently, we used a forward genetic screen in the recombinant inbred mouse set of BXDs (Loos et al., 2014), to question what factors control levels of inhibitory control. These mice form a genetic reference population with a fixed genome allowing assessment of genetic covariance in terms of behavior and modulation of transcriptional activity (Plomin et al., 1991; Williams et al., 2001; Peirce et al., 2004; Chesler et al., 2005). We measured inhibitory control by the 5 -choice serial reaction time task (5CSRTT), a task with face validity to the human continuous performance task, in which attention parameters and motor impulsivity can be assessed (Cole and Robbins, 1989; Puumala et al., 1996; Humby et al., 2005). We identified the impulsivity locus (Impu1) located on chromosome 14 around marker rs6197032, at 34.5-41.4 Mb (build 37, mm9) (McElroy et al., 1996; Lombardo et al., 2012; Loos et al., 2014). This locus harbors Neuregulin3 (Nrg3), a member of the neuregulin family, which is important for nervous system development as well as schizophrenia (Sachs et al., 2000; Barros et al., 2009). The fact that the C57BL/6J allele contributed to increased impulsivity, that C57BL/6J mice showed increased $\mathrm{Nrg} 3$ expression in the medial prefrontal cortex (mPFC), a region important for impulsivity, and that Neuregulins play an important role in central nervous system function and neuropsychiatric diseases, culminated in testing the effect of $\mathrm{Nrg} 3$ overexpression in the mPFC on impulsivity. As hypothesized, higher levels of Nrg3 resulted in higher levels of impulsive action, whereas loss of Nrg3 decreased impulsive responding (Palanza, 2001; Krishnan and Nestler, 2011; Loos et al., 2014; Zhu et al., 2014). Together this showed that regulating Nrg3 levels is key to impulsivity, in which miRNAs could play an important role.

With the realization that specific disease symptoms could occur in several diseases, analysis of intermediate traits, known as endophenotypes (Almasy and Blangero, 2001; de Geus et al., 2001; Loos et al., 2009), soon became a popular strategy to find genetic and molecular underpinning of complex phenotypes.
This strategy has the advantage that animal models, harboring a fraction of the complexity of the disease, could contribute to solve the puzzle. Co-occurrence of several of these endophenotypes in a model could point to a shared molecular mechanism. In this study we used a bioinformatics approach (Robbins et al., 2012; Mulligan et al., 2013) to question what behavioral effects are comorbid with the impulsivity trait (Loos et al., 2014), and what miRNAs could be driving levels of impulsivity. Therefore, we performed a comprehensive analysis to first select traits that are overrepresented with impulsivity, and second to select microRNAs of which the expression in amygdala is associated with impulsivity, using GeneNetwork data and several bioinformatic tools. We selected microRNAs based on three criteria; (1) their location within the Impul locus, (2) targeting of the Nrg3 mRNA, and (3) a correlated expression with impulsivity and comorbid traits. Together, we observed that miRNAs miR-190b, miR-28a, -219a, -340, and -491-5p may contribute to synaptic rearrangements and plasticity in the "impulsive" amygdala.

\section{METHODOLOGY \\ GENENETWORK CORRELATION ANALYSIS}

From the GeneNetwork database (http://www.genenetwork.org) (Rosen et al., 2007), we selected behavioral traits correlated with motor impulsivity (number of premature pokes; trait \#16311) as measured in the 5CSRTT (Loos et al., 2014) and performed the analysis in a two-step fashion: (1) we ranked correlations using Spearman rank test with n-number larger than 15 overlapping strains, as many traits in the database are from relatively small cohorts, and with corrected $P$-value $<0.01$; and $(2)$ we performed a trait overrepresentation test using key word searches, in which significantly correlated traits should be overrepresented in the GeneNetwork database. Key words representing the trait of interest were selected to preferably yield $\sim 50-100$ traits upon searching the GeneNetwork database, in order to get evenly sized groups based on this search.

For miRNA correlating with impulsivity, we focused on the amygdala because of its importance in emotional regulation, and because miRNA expression in BXD mice is well documented in the GeneNetwork database (http://www.genenetwork.org/dbdoc/INIA_AmgCoh_0311.html) with expression data available for $50 \mathrm{BXD}$ strains. Significant correlations were calculated for traits with $\geq 25$ strains in overlap. Correlation between traits or between traits and amygdala miRNAs is shown as the correlation coefficient (rho; Pearson product moment) with two-sided $P$-value indicated.

\section{microRNA VALIDATION}

Expression of miRNAs used for correlation analysis was based on Affymetrix probes (Table 3) of the GeneNetwork amygdala database (INIA Amygdala Cohort Affy MoGene 1.0ST (March 11 RMA). We validated each probe set specificity in detecting miRNA expression by alignment of probe sequences with the most current mouse genome browser and establishing their locations to miRNA genes. Location of these genes and sequence alignment of probe sets to these genes was further validated by miRBase, a free biological database that acts as an archive of all miRNA sequences and annotations (Griffiths-Jones, 2006; 
Griffiths-Jones et al., 2006), which also provides naming standards of microRNAs. Since recently miRNA nomenclature has changed (Arora et al., 2013), we matched GeneNetwork miRNA names with the most current miRNA names and used them throughout the paper (Table 3). Note also that GeneNetwork and miRBase are using different mouse genome assemblies, each providing different genomic coordinates for microRNA genes. Therefore, we used the NCBI Remap tool (http://www.ncbi.nlm. nih.gov/genome/tools/remap) to convert and match positions between assembly versions. Also, presence of SNPs in the regions targeted by probes can affect their efficacy, and may lead to false gene expression level results. We verified, using GeneNetwork variant browser, lack of SNPs in the genomic sequences targeted by these probes in both, $\mathrm{C} 57 \mathrm{Bl} / 6 \mathrm{~J}$ and $\mathrm{DBA} 2 / \mathrm{J}$ mice (Table 3).

\section{TARGETSCAN}

We used TargetScanMouse (Release 6.2) to predict mouse miR190b-5p targets. Among many miRNA target prediction software TargetScan seems to be very reliable (Friedman et al., 2009), and it uses two prediction scores. The aPCT score (a positive score) is based on the probability of aggregated preferentially conserved targeting, which is an indicator that a site is conserved due to selective maintenance of miRNA targeting rather than by chance. The total context (TC) score (a negative score) provides predictions based on four features of the putative microRNA-binding site: length of its complementarity to the microRNA's seed region (7-mer or 8-mer), position of the complementarity ( $3^{\prime}$-end), flanking of the binding site by adenosines, and a position within the 3'-UTR. To ensure high probability of interactions between miR-190b-5p and its targets and avoid false-positives we applied stringent criteria using simultaneously both scores $(\mathrm{aPCT}>0.2$; $\mathrm{CT}<-0.07$ ) thereby selecting the top $\sim 33 \%$ of targets (34 out of 103 total targets) with the highest aPCT score and the lowest TC score.

\section{GeneMania}

We used GeneMania to predict the miR-190b/Nrg3 interactive network. GeneMania is a freely available web interface designed to determine molecular interactions based on a set of input genes in different species (Warde-Farley et al., 2010). We originally input all 34 targets of miR-190b-5p using network weighting as "equal as determined by the network" (see Figures 4A,C), or using Gene Ontology (GO)-based weighting for "biological terms" (see Figures 4B,D) to maximize prediction of connectivity between all input genes. GeneMania provides eight interaction categories (co-expression, co-localization, physical interaction, genetic interaction, shared protein domains, pathway co-participation, predicted relationship and other) with ten to a hundred new genes shown. We restricted our output data to only ten new genes and three categories (co-expression, co-localization, physical interaction). We chose these three categories because they describe the most direct interactions between molecules. This approach allowed us to obtain a small network of highly interconnected genes with strong interactions centered on $\mathrm{Nrg} 3$. In a second round, 3 targets, and 4 related genes were used as input.
In addition, we used GeneMania to perform the functional enrichment analysis based on Gene Ontology (GO) terms augmented among genes in the network. Only biological terms with $P<0.05$ after correction for the false discovery rate (FDR) were considered to be enriched. We further used DAVID (The Database for Annotation, Visualization and Integrated Discovery) (Huang et al., 2007) and GeneCards (Rebhan et al., 1997) to validate GeneMania results describing association of members of the Nrg3-network with specific functional terms.

\section{mirPath}

DIANA mirPath (Maragkakis et al., 2009) is a freely available web interface designated by DIANA (DNA Intelligent Analysis) Laboratory to estimate the impact miRNA effects on biological pathways. Both the effect of a single miRNA and the combinatorial effects of multiple miRNAs are available. After performing an enrichment analysis mirPath marks positions of miRNA-targeted genes in biological pathways provided by Kyoto Encyclopedia of Genes and Genomes (KEGG) (Kanehisa and Goto, 2000). The KEGG Pathway database is the most comprehensive resource of interactions of gene products and provides wiring diagrams of interaction networks. DIANA mirPath output consists of KEGG pathways sorted according to $P$ value after correction for the false discovery rate (FDR, Hochberg and Benjamini, 1990). Only pathways with $P<0.05$ were considered to be enriched. Lower $P$-value indicates bigger biological impact of miRNA(s) on that pathway.

\section{RESULTS CORRELATIONS OF IMPULSIVITY WITH OTHER BEHAVIORAL TRAITS}

Impulsivity, measured as the lack of inhibitory control in 5CSRTT (Loos et al., 2012, 2014), is a characteristic of many psychiatric diseases (e.g., ADHD, schizophrenia, depression, addiction), each represented by complex interactions of several traits. To understand the role of miRNAs in impulsivity, we first assessed whether impulsive behavior would correlate with other phenotypic traits, as this could be an indication that the genetic makeup of BXD strains controlling impulsivity play a role in other traits as well. Using the GeneNetwork database, we performed the analysis in a two-step fashion: (1) we ranked correlations using Spearman rank test with n-numbers larger than 15 overlapping strains, and with $P$-values $<0.01$; and (2) we performed a trait overrepresentation test using key word searches, in which significantly correlated traits should be overrepresented in the GeneNetwork database. This approach should prevent finding of a correlation by pure chance, albeit that there still could be a bias toward studies with more in depth phenotyping. In total, we selected 34 traits (Table 1, Figure 1). From these 34 traits, 17 compound traits were selected using a Fischer's exact test (Table 2, Figure 1). Three of these traits ("Attention/Cognition," "Depression/Immobility," "Metabolism/Body weight gain") were overrepresented showing a significant correlation $(P<0.05)$ with impulsivity, whereas one trait ("Anxiety/Novel open field") showed a trend $(P<0.10)$ toward overrepresentation. Although classical parameters of an open field relate to anxiety, the correlated traits were mostly related to rearing behavior. 
Table 1 | Correlation of phenotypic traits with impulsivity in BXD mouse strains.

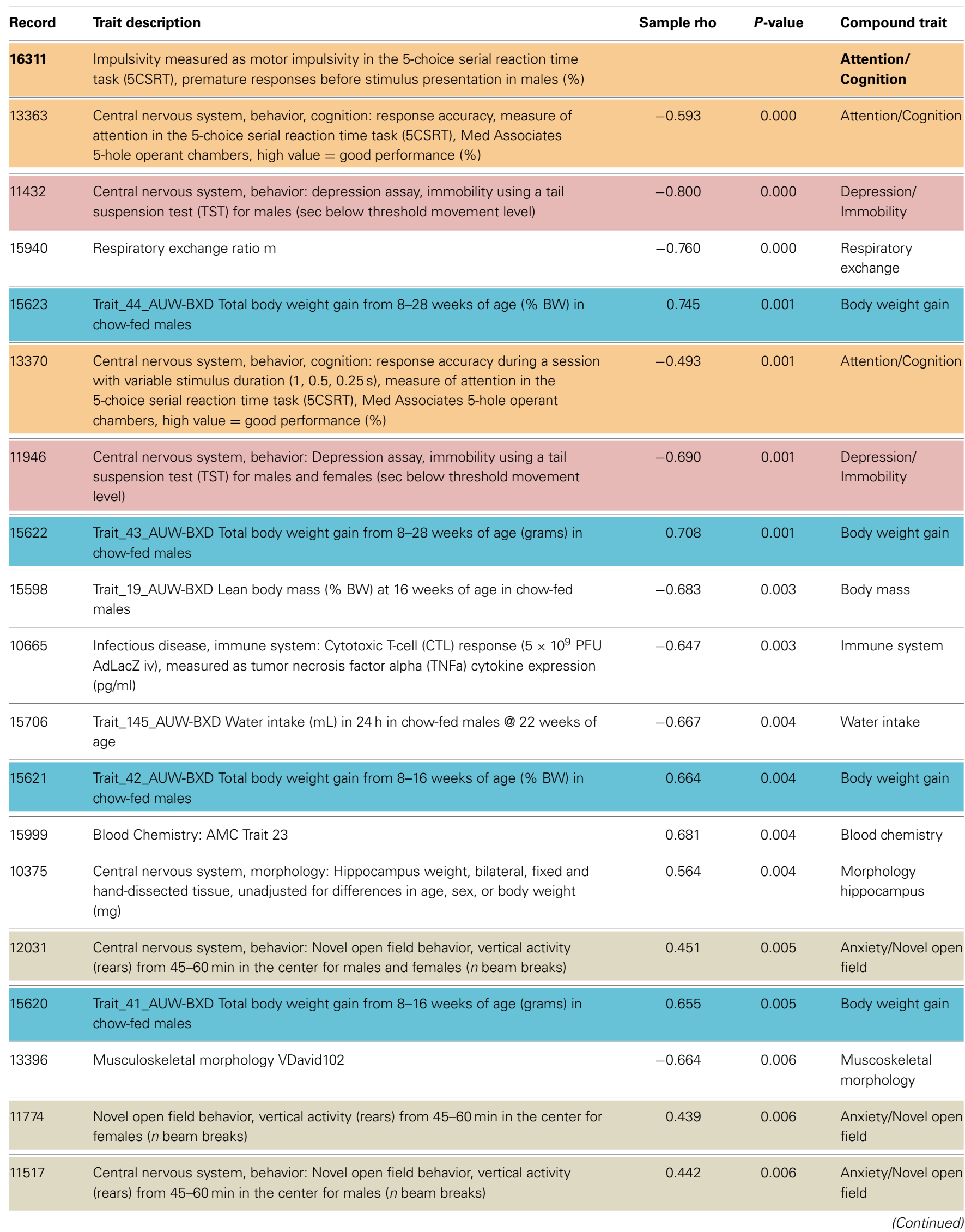


Table 1 | Continued

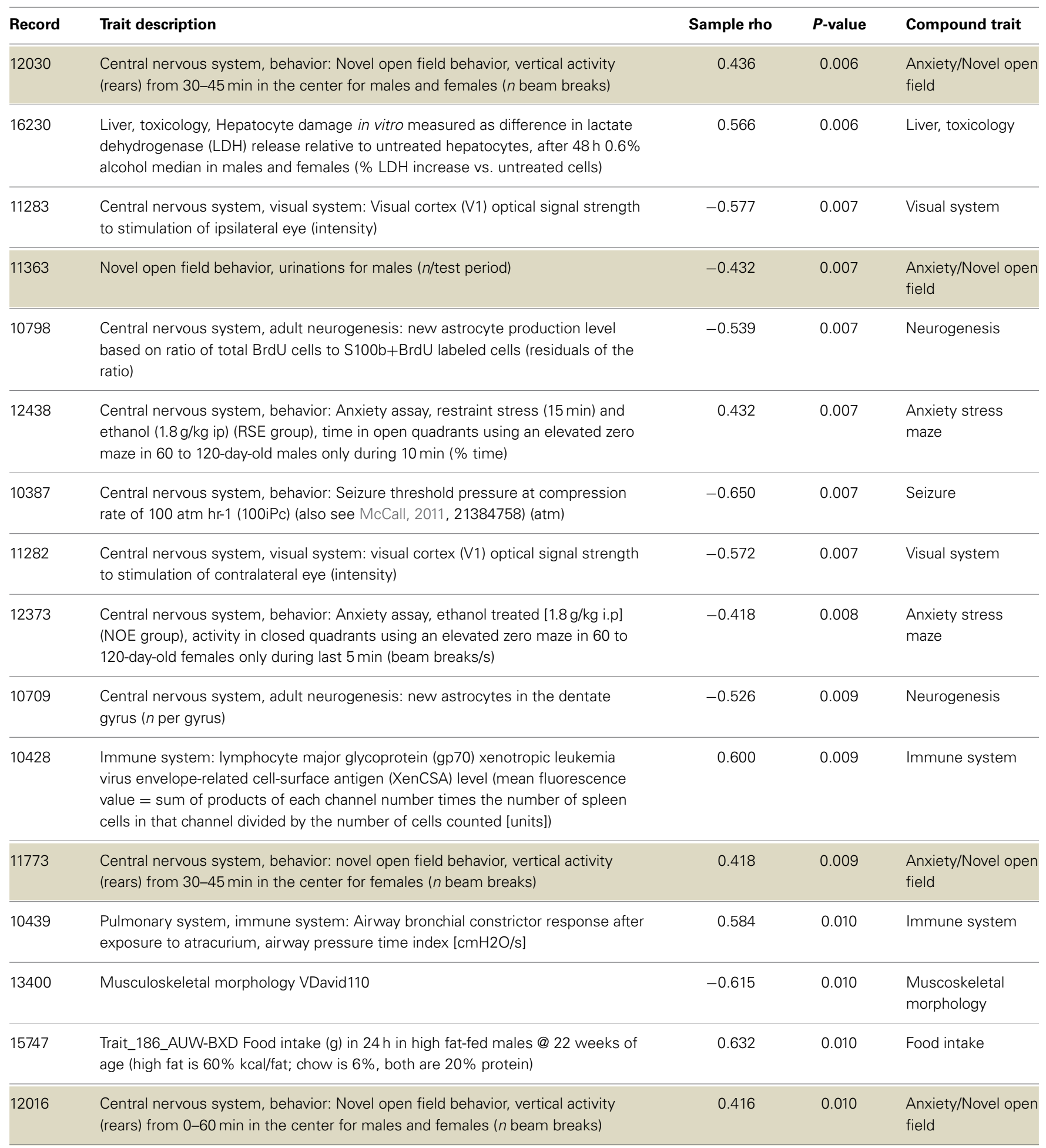

The impulsivity trait (16311; bold) was correlated with all available phenotypic traits using GeneNetwork. Traits of the highest significant correlations ( $P<0.001)$ for $n=15$ BXD strains are displayed with their Spearman rank correlation coefficient (Sample rho) sorted by the P-value. Compound traits are color-coded when overrepresented (see Table 2). 


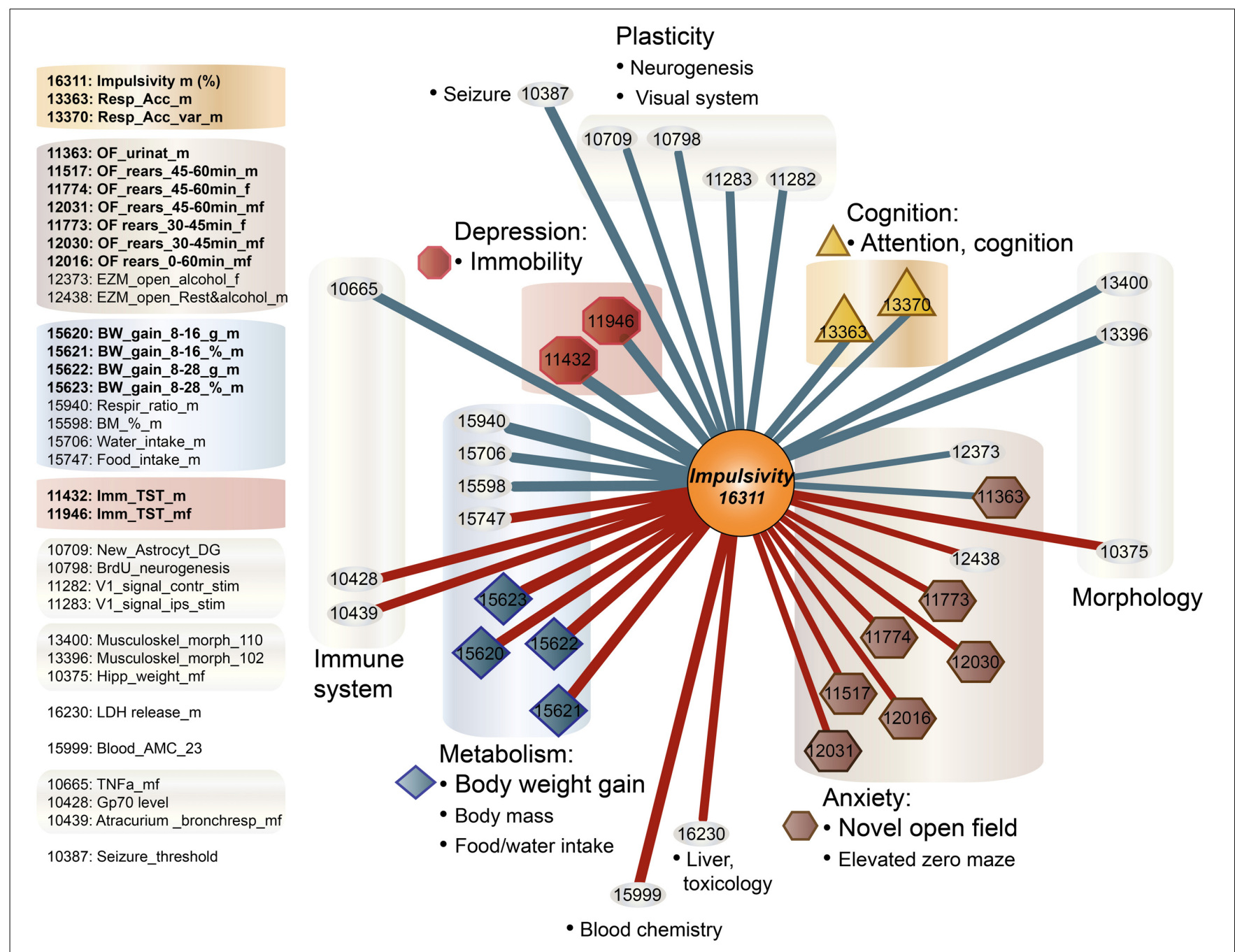

FIGURE 1 | Correlation of traits with impulsivity in BXD strains. Individual behavioral traits and their compound traits (colored) are shown. Traits number IDs are taken from the GeneNetwork records. The impulsivity trait (ID\#:16311) is placed in the center of the figure. Color of lines indicates the direction of the correlation (blue, negative; red, positive), and line thickness corresponds to the size of the Spearman rank correlation (thicker line means stronger correlation).

\section{SELECTION AND CORRELATION OF microRNAs WITH IMPULSIVITY AND COMPOUND TRAITS}

The amygdala is involved in emotional regulation of impulsive behavior, and miRNA expression in this brain region of the BXD mice is well documented in the GeneNetwork database. Therefore, we used this miRNA expression dataset to determine which miRNA correlates with impulsivity and its overrepresented traits. In addition, two traits (\#15598, \#15747) were added to the "Body weight gain" compound trait, as they relate to body weight and food intake, respectively. In our analysis we used a comprehensive, three-prong approach of miRNA selection.

First, we selected a miRNA located within a recently established impulsivity locus located on chromosome 14 in mice (Loos et al., 2014) (Figure 2A). Despite a substantial size of this locus $(6.9 \mathrm{Mb})$, it contains only a single miRNA gene (chr14:34,894,609-34,894,706) called mir-346 (Figure 2B). A product of the mir-346 gene is a hairpin-shaped mir-346 precursor, which gives rise to two mature miRNAs: miR-346-5p and
miR-346-3p (Figure 2C), of which the former is a predominant form. Expression of the miR-346-5p miRNA in BXD mice was not correlated with impulsivity (Table 3), and therefore was not included into further analyses.

Second, due to our previous observation of a significant association between $\mathrm{Nrg} 3$ gene expression and the lack of inhibitory control, or impulsive action (Loos et al., 2014), we decided to determine which miRNAs target murine Nrg3 mRNAs. Based on the mouse genome browser, the $N r g 3$ gene produces three distinct mRNAs, each with a different $3^{\prime}$-UTR length (Figure 2D). We observed that two of these mRNAs have very short $3^{\prime}$ UTRs ( $56 \mathrm{nts}$ ) that do not possess any miRNA binding sites. In contrast, the NM_001190187 mRNA contains a substantially longer $3^{\prime}$-UTR (1582 nts) with five different miRNA binding sites grouped into two clusters (Figure 2E). The miRNAs binding to these sites, i.e., miR-137-3p, miR-155a-5p, miR-190b-5p, miR-204-5p, miR-30b-5p, miR-30c-5p, miR-30d-5p, miR-30e$5 p, \operatorname{miR}-384-3 p$, were selected for the correlation analysis with 
Table 2 | Selection of overrepresented compound traits correlated with the impulsivity trait (16311).

\begin{tabular}{|c|c|c|c|c|}
\hline Compound trait & \#Correlated traits & Total traits in GeneNetwork & Representation ratio & $P$-value \\
\hline Attention, cognition & 2 & 9 & 12.09 & 0.0181 \\
\hline Blood chemistry & 1 & 345 & 0.16 & 0.0330 \\
\hline Immune system & 3 & 499 & 0.33 & 0.0443 \\
\hline Anxiety/Novel open field & 7 & 179 & 2.13 & 0.0942 \\
\hline Food intake & 1 & 18 & 3.02 & 0.3045 \\
\hline Muscoskeletal morphology & 2 & 276 & 0.39 & 0.3076 \\
\hline Seizure & 1 & 29 & 1.88 & 0.4354 \\
\hline Body mass & 1 & 30 & 1.81 & 0.4460 \\
\hline Hippocampal morphology & 1 & 48 & 1.13 & 0.6050 \\
\hline Total & 34 & 1850 & & \\
\hline
\end{tabular}

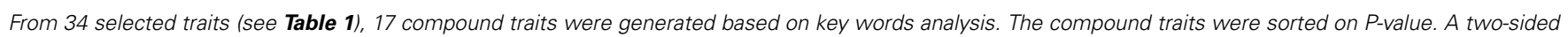

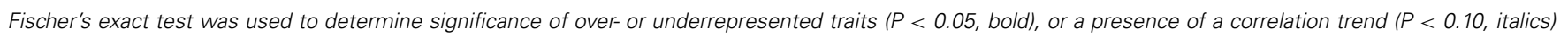

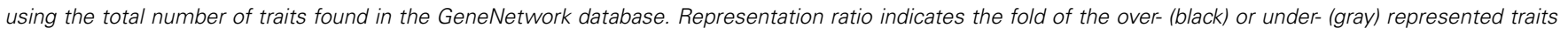
compared with the original query.

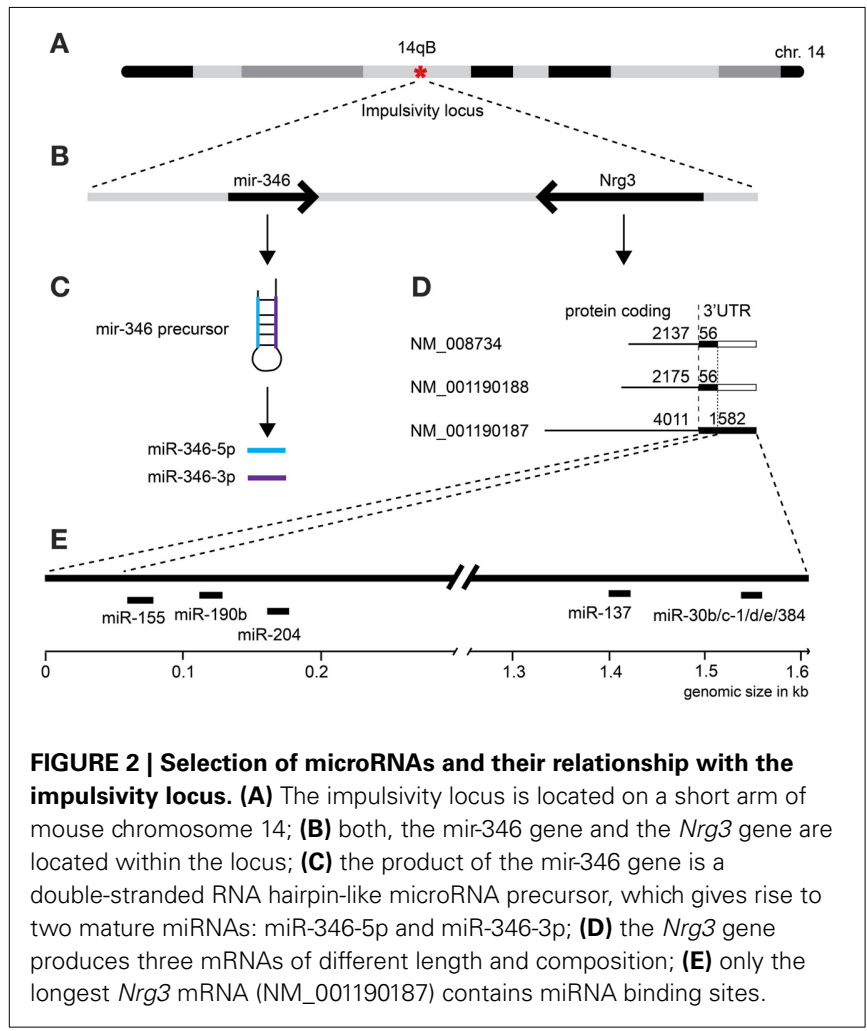

impulsivity. We established that only miR-190b-5p is significantly correlated with impulsive action and that this correlation is negative, meaning that with increased impulsivity of BXD mice the expression of miR-190b-5p in the amygdala is lowered.
We further determined correlation of miR-190b-5p with the overrepresented compound traits and only observed significant correlations with two metabolic traits (Table 4, Figure 3).

Finally, we established that expression of miR-28a-5p, miR219a-5p, miR-340-5p, and miR-491-5p, although not directly related to the impulsivity locus or $\mathrm{Nrg} 3$, is significantly correlated with the impulsivity trait (Table 3 ). Three of these miRNAs (miR28a-5p, miR-340-5p, and miR-491-5p) were negatively correlated with impulsivity, whereas expression of miR-219a-5p showed a positive correlation. Furthermore, the expression of this set of miRNAs was assessed for correlated expression with the overrepresented compound traits. The expression of miR-340-5p was strongly and positively correlated with all anxiety traits, most of which represented rearing behavior (Table 4, Figure 3). Rearing could probably represent phenotypes of vigilance or learning (Görisch and Schwarting, 2006). Also, expression of miR-491$5 p$ was weakly positively correlated with these rearing traits. Consistent with the positive correlation of miR-28a-5p with impulsivity, it showed a negative correlation with the attention traits of the 5CSRTT (Table 4, Figure 3). However, expression of miR-219a-5p was not correlated with any other trait (Table 4, Figure 3).

\section{PLAUSIBLE ROLE OF CORRELATED microRNAs IN NEURONAL FUNCTION}

As a next step in identifying putative regulators of impulsivity and overrepresented compound traits in the amygdala, we attempted to better understand their role in neuronal function. Because a single miRNA typically regulates several protein-coding mRNAs we first established which gene products are, in addition to Nrg3, miR-190b-5p targets. Subsequently, to determine 


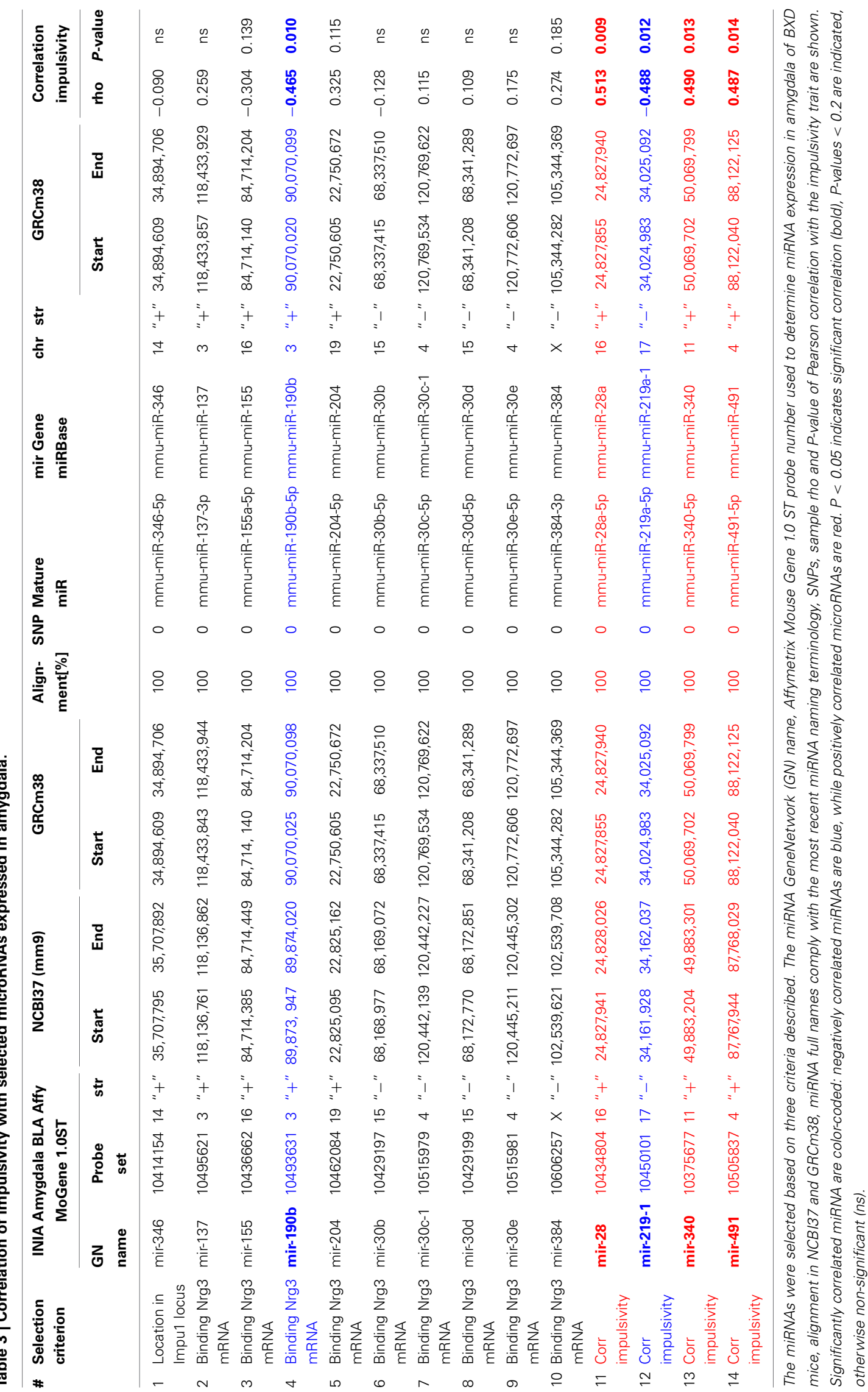


Table 4 | Correlation between overrepresented compound traits and selected microRNAs expressed in amygdala.

\begin{tabular}{|c|c|c|c|c|c|c|c|c|c|c|c|}
\hline Trait \# & \multicolumn{2}{|c|}{ mir-190b } & \multicolumn{2}{|c|}{ mir-28 } & \multicolumn{2}{|c|}{ mir-219-1 } & \multicolumn{2}{|c|}{ mir-340 } & \multicolumn{2}{|c|}{ mir-491 } & $n$ \\
\hline 16311 & 0.465 & 0.019 & 0.513 & 0.009 & -0.488 & 0.013 & 0.490 & 0.013 & 0.487 & 0.014 & 25 \\
\hline 13363 & 0.083 & ns & -0.510 & 0.008 & 0.257 & ns & -0.002 & ns & -0.076 & ns & 26 \\
\hline 13370 & 0.120 & ns & -0.476 & 0.014 & 0.223 & ns & -0.066 & ns & -0.096 & ns & 26 \\
\hline 11363 & 0.240 & 0.136 & -0.158 & ns & 0.132 & ns & -0.156 & ns & -0.297 & 0.063 & 40 \\
\hline 11517 & -0.130 & ns & 0.182 & ns & -0.136 & ns & 0.408 & 0.010 & 0.241 & 0.139 & 39 \\
\hline 11773 & -0.181 & ns & 0.002 & ns & -0.016 & ns & 0.507 & 0.001 & 0.288 & 0.072 & 40 \\
\hline 11774 & -0.154 & ns & 0.026 & ns & 0.029 & ns & 0.439 & 0.005 & 0.300 & 0.060 & 40 \\
\hline 12016 & -0.171 & ns & 0.031 & ns & -0.031 & ns & 0.480 & 0.002 & 0.271 & 0.090 & 40 \\
\hline 12030 & -0.177 & ns & 0.080 & ns & -0.059 & ns & 0.476 & 0.002 & 0.280 & 0.080 & 40 \\
\hline 15621 & -0.336 & 0.052 & 0.294 & 0.091 & -0.174 & ns & 0.182 & ns & 0.015 & ns & 34 \\
\hline 15622 & -0.155 & ns & -0.011 & ns & -0.272 & ns & 0.077 & ns & 0.127 & ns & 34 \\
\hline 15623 & -0.184 & ns & 0.059 & ns & -0.089 & ns & 0.028 & ns & 0.154 & ns & 34 \\
\hline 15598 & 0.375 & 0.029 & -0.106 & ns & -0.061 & ns & -0.269 & 0.124 & -0.317 & 0.068 & 34 \\
\hline 15747 & -0.351 & 0.042 & 0.394 & 0.021 & 0.354 & 0.040 & 0.350 & 0.042 & 0.274 & 0.117 & 34 \\
\hline
\end{tabular}

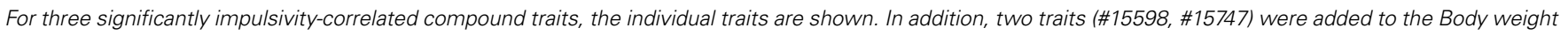

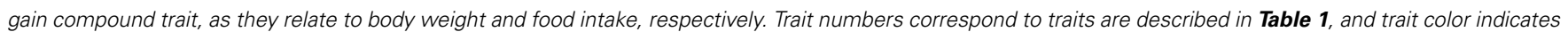
the correlation with impulsivity (red, positive; blue negative) Sample rho and P-value of Pearson correlation ( $n \geq 25$ strains), as well as well as $n$-number in overlap,

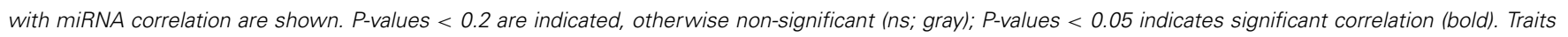
with $n<25$ strains in overlap with miRNA expression are not shown.

the Nrg3-related network of miR-190b-5p targets, we predicted biological interactions of these gene products with $\mathrm{Nrg3}$, as well as their functional importance using TargetScanMouse, GeneMania and DAVID (Huang et al., 2007; Friedman et al., 2009; WardeFarley et al., 2010), respectively.

We applied stringent TargetScan conditions and selected only targets with simultaneously a high aPCT score and a low total context score (top 33\% of all targets; see Methodology). This approach, ensuring selection of high probability interactions between miR-190b-5p and its targets, yielded 34 genes (Table 5). In order to view genes that are related to the Nrg3 network, the 34 selected targets underwent an interaction network analysis using GeneMania, in which we only selected gene products directly linked to Nrg3, based on evidence indicating co-localization, co-expression and physical interaction with $\mathrm{Nrg} 3$, for different network weighting (Figures 4A,B). Besides three miR-190b-5p targets (Myo5a, Celf6, and Nlgn1) interacting directly with Nrg3, we also discovered additional gene products as members of the Nrg3 network, which were ErbB4, the receptor of Nrg3, and Grlb, the b-subunit of the glycine receptor (Figure 4A). In addition, the adhesion molecule Nrxn1, and the complement component C1ql3 (Figure 4B) were detected. In order to evaluate an Nrg3-network of closely interacting gene products, we took these eight genes for a second round of establishing gene interactions (Figures 4C,D). Relationships of each gene with a particular biological term within each process are shown in Table 6. It appears that this network contributes mostly to three biological processes, all of them pertinent to the neuronal (synaptic) function (Figures 4C,D). Thus, miR-190b-5p, via its targets and genes within the Nrg3-network, seems to control synaptic activity.

For the other miRNAs, miR-28a-5p, miR-219a-5p, miR-340$5 \mathrm{p}$, and miR-491-5p, which were significantly correlated with the impulsivity trait (Table 3 ), the expression of three (miR-28a$5 p$, miR-340-5p, and miR-491-5p) was correlated in the same direction (positive correlation) with the impulsivity trait, whereas the expression of miR-219a-5p was correlated in the opposite direction. Typically, miRNAs work as gene product suppressors; increased expression of a miRNA decreases expression of its targets, while decreased expression of a miRNA increases expression of its targets. To understand the contribution of these miRNAs to biological processes, we uploaded all three positively correlated microRNAs as a one group and the negatively correlated microRNA (miR-219a-5p) as a second group into mirPath v2.0 (Vlachos et al., 2012). This program allows for determination of interactions of pathways regulated by multiple miRNAs or a single miRNA. The simultaneous input of all 4 miRNAs, although possible, would make it difficult to distinguish down-regulated from up-regulated effects of miRNAs on their targets. The top five of enriched, neuronal function-related pathways are shown in Table 7 (upper part) with the axonal guidance pathway being regulated most. We next determined in a similar fashion pathways regulated by miR-219a-5p, a brain-specific miRNA. Axonal 


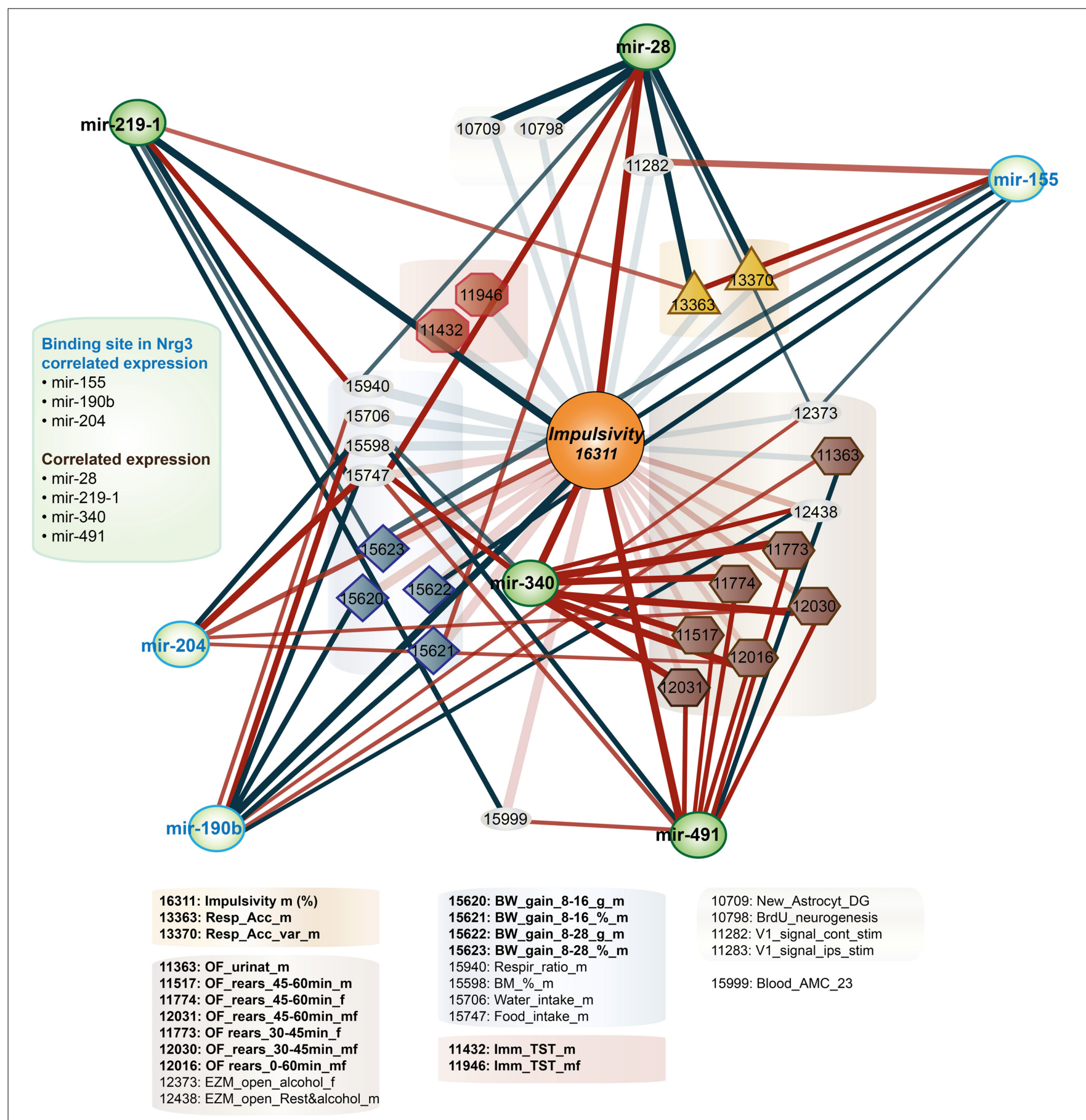

FIGURE 3 | Impulsivity-related microRNA expression network in amygdala. Amygdala miRNAs (green circles) of which the expression is correlated with impulsivity (lack of inhibitory control) (Table 3) are shown. Correlation of miRNAs with impulsivity and compound traits are shown as lines. Colors of lines indicate the direction of the correlation (blue, negative; red, positive), intensity of the color depicts $P$-value of Pearson correlation (one sided: dark, $P<0.05$; light, $P<0.10$ ) and line thickness corresponds to the size of the Pearson rank correlation (thicker line means stronger correlation). This network is overlaid on the impulsivity correlation trait network from Figure 1 (faded colors) to show relationship of traits and miRNAs. For the GeneNetwork IDs of individual traits see Table $\mathbf{1}$. guidance also appeared to be one of the pathways targeted by miR-219a-5p (Table 7, lower part). Since miR-219a-5p is correlated with impulsivity in an opposite direction as the three other miRNAs, it could attenuate some of the effect of these
miRNAs. Figure 5 shows the axonal guidance pathway with gene products targeted by specific miRNAs used in this study. Axonal guidance is controlled by several guidance cues (netrins, ephrins, slits and semaphorins), which affect axonal attraction, repulsion 
Table 5 | Targets of miR-190b.

\begin{tabular}{|c|c|c|c|c|c|}
\hline \# & Gene symbol & mRNA accession \# & Gene full name & aPCT score & TC score \\
\hline 1 & Dennd5b & NM_177192 & DENN/MADD domain containing 5B & 0.44 & -0.42 \\
\hline 2 & Dmd & NM_007868 & Dystrophin, muscular dystrophy & 0.44 & -0.33 \\
\hline 3 & Tnrc6b & NM_144812 & Trinucleotide repeat containing $6 \mathrm{~b}$ & 0.44 & -0.31 \\
\hline 4 & Kcnq5 & NM_001160139 & Potassium voltage-gated channel, subfamily $\mathrm{Q}$, member 5 & 0.44 & -0.30 \\
\hline 5 & Irs4 & NM_010572 & Insulin receptor substrate 4 & 0.43 & -0.28 \\
\hline 6 & Trp53inp1 & NM_001199105 & Transformation related protein 53 inducible nuclear protein 1 & 0.42 & -0.27 \\
\hline 7 & Epc2 & NM_172663 & Enhancer of polycomb homolog 2 (Drosophila) & 0.40 & -0.37 \\
\hline 8 & Gphn & NM_145965 & Gephyrin & 0.40 & -0.27 \\
\hline 9 & Celf4 & NM_001146292 & CUGBP, Elav-like family member 4 & 0.40 & -0.21 \\
\hline 10 & Fgf14 & NM_010201 & Fibroblast growth factor 14 & 0.40 & -0.16 \\
\hline 11 & Setbp1 & NM_053099 & SET binding protein 1 & 0.37 & -0.26 \\
\hline 12 & Tnrc6a & NM_144925 & Trinucleotide repeat containing $6 a$ & 0.35 & -0.31 \\
\hline 13 & Myo5a & NM_010864 & myosin 5A & 0.30 & -0.36 \\
\hline 14 & Samd4 & NM_001037221 & Sterile alpha motif domain containing 4 & 0.30 & -0.28 \\
\hline 15 & Foxp2 & NM_053242 & Forkhead box P2 & 0.30 & -0.21 \\
\hline 16 & Tnrc6c & NM_198022 & Trinucleotide repeat containing 6C & 0.27 & -0.22 \\
\hline 17 & Bai3 & NM_175642 & Brain-specific angiogenesis inhibitor 3 & 0.24 & -0.34 \\
\hline 18 & Stc1 & NM_009285 & Stanniocalcin 1 & 0.23 & -0.45 \\
\hline 19 & Nlgn1 & NM_001163387 & Neuroligin 1 & 0.23 & -0.40 \\
\hline 20 & Arpc5 & NM_026369 & Actin related protein $2 / 3$ complex, subunit 5 & 0.22 & -0.35 \\
\hline 21 & Fndc3b & NM_173182 & Fibronectin type III domain containing 3B & 0.22 & -0.25 \\
\hline 22 & Slco5a1 & NM_172841 & Solute carrier organic anion transporter family, member $5 \mathrm{~A} 1$ & 0.22 & -0.24 \\
\hline 23 & Panx2 & NM_001002005 & Pannexin 2 & 0.22 & -0.13 \\
\hline 24 & Slc30a4 & NM_011774 & solute carrier family 30 (zinc transporter), Member 4 & 0.22 & -0.11 \\
\hline 25 & Agfg1 & NM_010472 & ArfGAP with FG repeats 1 & 0.22 & -0.11 \\
\hline 26 & Mfap3l & NM_001177881 & Microfibrillar-associated protein 3-like & 0.22 & -0.10 \\
\hline 27 & Anxa7 & NM_001110794 & Annexin A7 & 0.22 & -0.10 \\
\hline 28 & Celf6 & NM_175235 & CUGBP, Elav-like family member 6 & 0.22 & -0.10 \\
\hline 29 & Ubr2 & NM_001177374 & Ubiquitin protein ligase E3 component n-recognin 2 & 0.22 & -0.09 \\
\hline 30 & Cramp1l & NM_020608 & Crm, cramped-like (Drosophila) & 0.22 & -0.09 \\
\hline 31 & Nrg3 & NM_001190187 & Neuregulin 3 & 0.22 & -0.08 \\
\hline 32 & Man2a1 & NM_008549 & Mannosidase 2, alpha 1 & 0.22 & -0.08 \\
\hline 33 & Tbc1d14 & NM_001113362 & TBC1 domain family, member 14 & 0.21 & -0.32 \\
\hline 34 & Tcf4 & NM_001083967 & mRNAion factor 4 & 0.21 & -0.24 \\
\hline
\end{tabular}

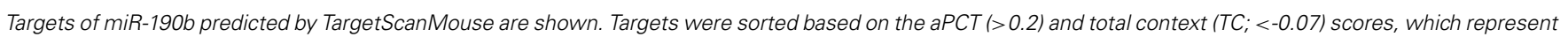

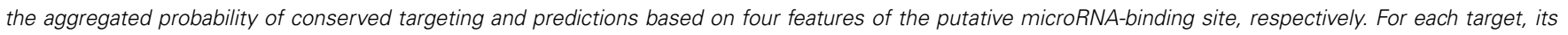
symbol, a representative $m R N A$, and full name of the gene from which the target is produced are shown.

and outgrowth. The effect of miR-219a-5p seems to be small and mainly limited to slits-related guidance cues and (partially) semaphorins cues, whereas the netrins and ephrins cues were affected exclusively by the positively correlated miRNAs (miR28a-5p, miR-340-5p, and miR-491-5p). Thus, the combinatorial effect of all miRNAs correlated with impulsivity on the KEGG axonal guidance pathway is carried out mostly by the miRNAs that are positively correlated with impulsivity (Figure 5). Considering that positive correlations mean that an increase in impulsivity is associated with an increase in miRNA expression in the amygdala, and since the main action of miRNAs is suppression of its targets, we concluded that the miRNAs correlating with impulsivity most likely attenuate axon repulsion in the amygdala (Figure 5).
Two other pathways were commonly regulated by both the positively- and the negatively-correlated miRNAs. These were Wnt signaling and endocytosis (Table 7). Similarly to the axonal guidance, both of these pathways seem to be more affected by the positively correlated miRNAs (miR-28a-5p, miR-340$5 p$, and miR-491-5p) rather then the negatively correlated one (miR-219a-5p), of which the effect on these pathways is barely significant (Table 7).

In summary, our comprehensive bioinformatic analysis seems to indicate that miRNAs in the amygdala may contribute to the development of the impulsivity trait. On one hand, miR-190b-5p seems to control synaptic activity, whereas other miRNAs (primarily miR-340-5p) may control axonal guidance. 

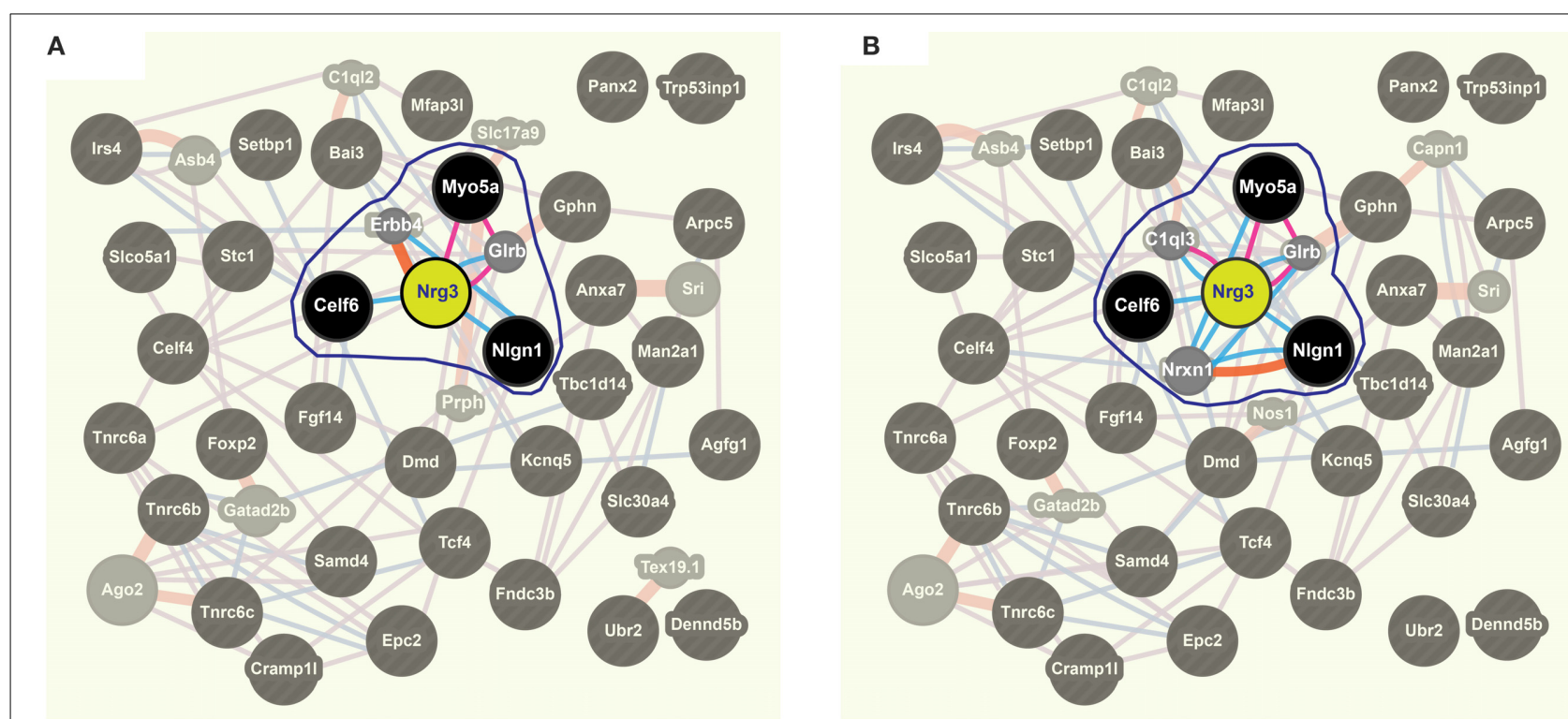

C

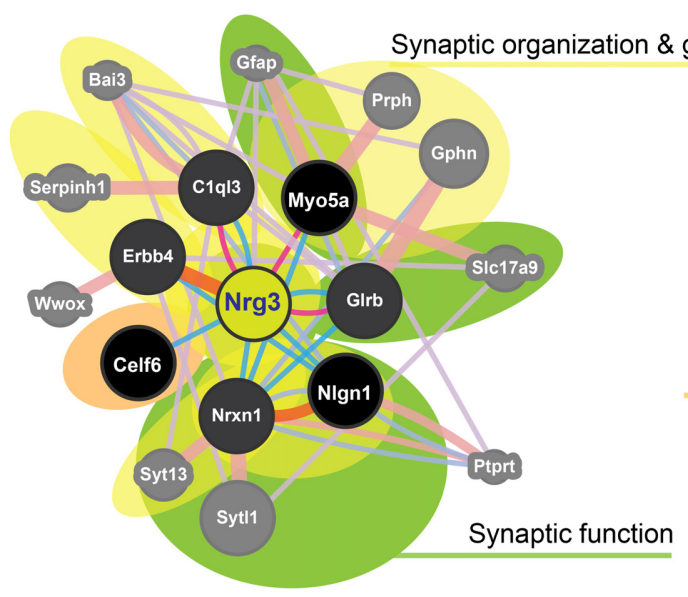

FIGURE 4 | Impulsivity-related miR-190b and Nrg3 expression network in amygdala. (A,B) Networks of reciprocal interactions of 34 targets (black) of Nrg3 selected based on GeneMania stringent interaction criteria (for full names see Table 5), weighted equally by network (a), or weighted for biological process (B). A maximum of 10 additional genes is shown (gray). Highlighted (encircled) are gene products and their interactions directly linked to Nrg3. (C,D) Nrg3 network of closely interacting gene products, with direct

\section{DISCUSSION}

Impulsive action, the tendency to act "on a spot" without a foresight and the inability to inhibit such a prepotent response, is a complex behavioral trait. This type of impulsivity can be advantageous in situations requiring a rapid response, but often is detrimental and associated with psychiatric disorders. Impulsivity is moderately heritable (Kuntsi et al., 2006; Schachar et al., 2011; Crosbie et al., 2013), which suggests partial genetic etiology (Bevilacqua and Goldman, 2013). We have recently established the presence of a genetic locus for motor impulsivity in the genetic resource of $\mathrm{BXD}$ strains demonstrating an important role for Nrg3 in the mPFC (Loos et al., 2014).

Here, we observed that in $\mathrm{BXD}$ mice the impulsivity trait is significantly associated with traits relevant to psychiatric
D

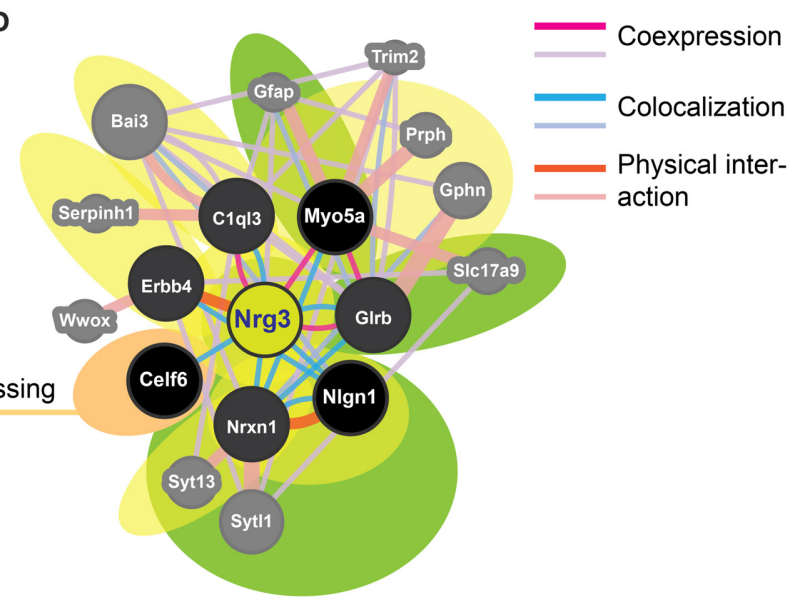

miR-190b-5p targets (black) and associated genes (dark gray) (see A,B) and their contribution to gene ontology (GO) biological processes in the Nrg3-network (color-coded: yellow, synaptic organization and genesis; green, synaptic function; orange, mRNA processing). A legend describing interaction categories is shown. Link thickness corresponds to strength (weight) of the interaction, and size of shape (gray only) indicates the weight of the interaction in the network. disorders: attention, depression, metabolism and forms of anxiety. Indeed, impulsive behavior is augmented in several psychiatric disorders, of which the most well-known is ADHD, in which it is one of the DSM-V criteria (American Psychiatric Association). The negative correlations of impulsivity with attention parameters, such as the $\%$ of correct responses as found here (Figure 1), are well-known and have been previously observed in different strains or pertubations (Loos et al., 2009; Counotte et al., 2011; Agnoli and Carli, 2012). A concerted, balanced relationship between attention and behavioral inhibition is central to the executive function model and effective adaptation to an ever-changing environment (Bari and Robbins, 2013).

In addition, impulsivity is often observed in bipolar patients, and co-occurs with periods of mania (McElroy et al., 1996; 


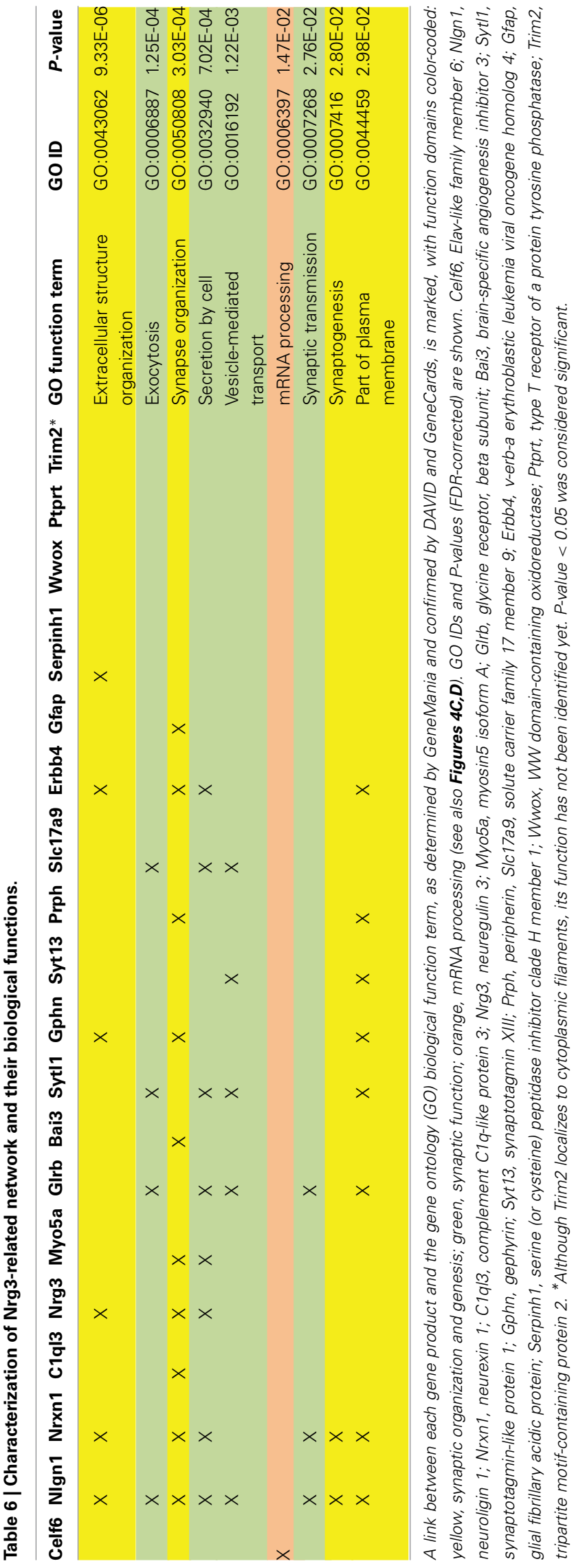

Table 7 | Biological pathways regulated by microRNAs correlated with impulsivity trait.

\begin{tabular}{ll}
\hline KEGG biological pathway & $\boldsymbol{P}$-value \\
\hline miR-28a, miR-340, and miR-491 & \\
Axon guidance & $2.18 \mathrm{E}-09$ \\
Wnt signaling pathway & $1.46 \mathrm{E}-07$ \\
Endocytosis & $2.38 \mathrm{E}-07$ \\
MAPK signaling pathway & $1.28 \mathrm{E}-05$ \\
Focal adhesion & $1.40 \mathrm{E}-05$ \\
miR-219a & \\
Endocytosis & $1.45 \mathrm{E}-02$ \\
Circadian rhythm & $2.81 \mathrm{E}-02$ \\
Axon guidance & $3.34 \mathrm{E}-02$ \\
Wnt signaling pathway & $4.73 \mathrm{E}-02$ \\
\hline
\end{tabular}

Enriched pathways related to neuronal functions are shown. Contribution of miR-28a-5p, miR-340-5p and miR-491-5p was assessed simultaneously due to their synchronized positive correlation. We have analyzed miR-219a separately because it was the only miRs correlated negatively with impulsivity. Analysis was performed using DIANA mirPath algorithm and KEGG software with FDR correction. P-value $<0.05$ was considered significant.

Lombardo et al., 2012). Interestingly, a positive family history of bipolar disorder could indicate an elevated risk to develop bipolar disorder in children with ADHD (Sachs et al., 2000). Many animal models of depression try to capture aspects of the disease in the anxiety domain, with classical anxiety tests, such as response to novelty in an open field or elevated plus maze, and test that assess helplessness and a low effort to escape, such as the tail suspension test and forced swim test (Palanza, 2001; Krishnan and Nestler, 2011; Zhu et al., 2014). Immobility in a tail suspension test (traits \#11946, \#11432), serving as proxy for depression, was negatively correlated with impulsivity. Although this is of interest from a clinical perspective, motor impulsivity could easily be confused with symptoms of hyperactivity in preclinical models. However, neither in our dataset, nor in the BXD dataset in GeneNetwork, locomotor activity in an open field is correlated with our impulsivity trait. This apparent lack of a correlation between impulsivity and activity in response to novelty has been observed before (Loos et al., 2009). Therefore, the correlation between impulsivity and immobility could indicate a shared biological mechanism.

Whereas motor impulsivity deals with acting before thinking despite possible adverse consequences, compulsivity entails actions that are persistently repeated despite adverse consequences (Robbins et al., 2012). Both psychological constructs have been hypothesized to result from failures of response inhibition or "top-down" cognitive control with overlapping mechanisms and brain areas involved (Wolters et al., 2008; Robbins et al., 2012; Callesen et al., 2013). Compulsivity is conceived as maladaptive behavior contributing to drug-taking, or excessive eating. In this respect, the positive correlation between impulsivity and several metabolism traits (Figure 1) in BXD mice may not come as a surprise. Recent studies showed that impulsivity scores in humans are associated with additive consumption of food and subsequently high BMI (Murphy et al., 2013). This 


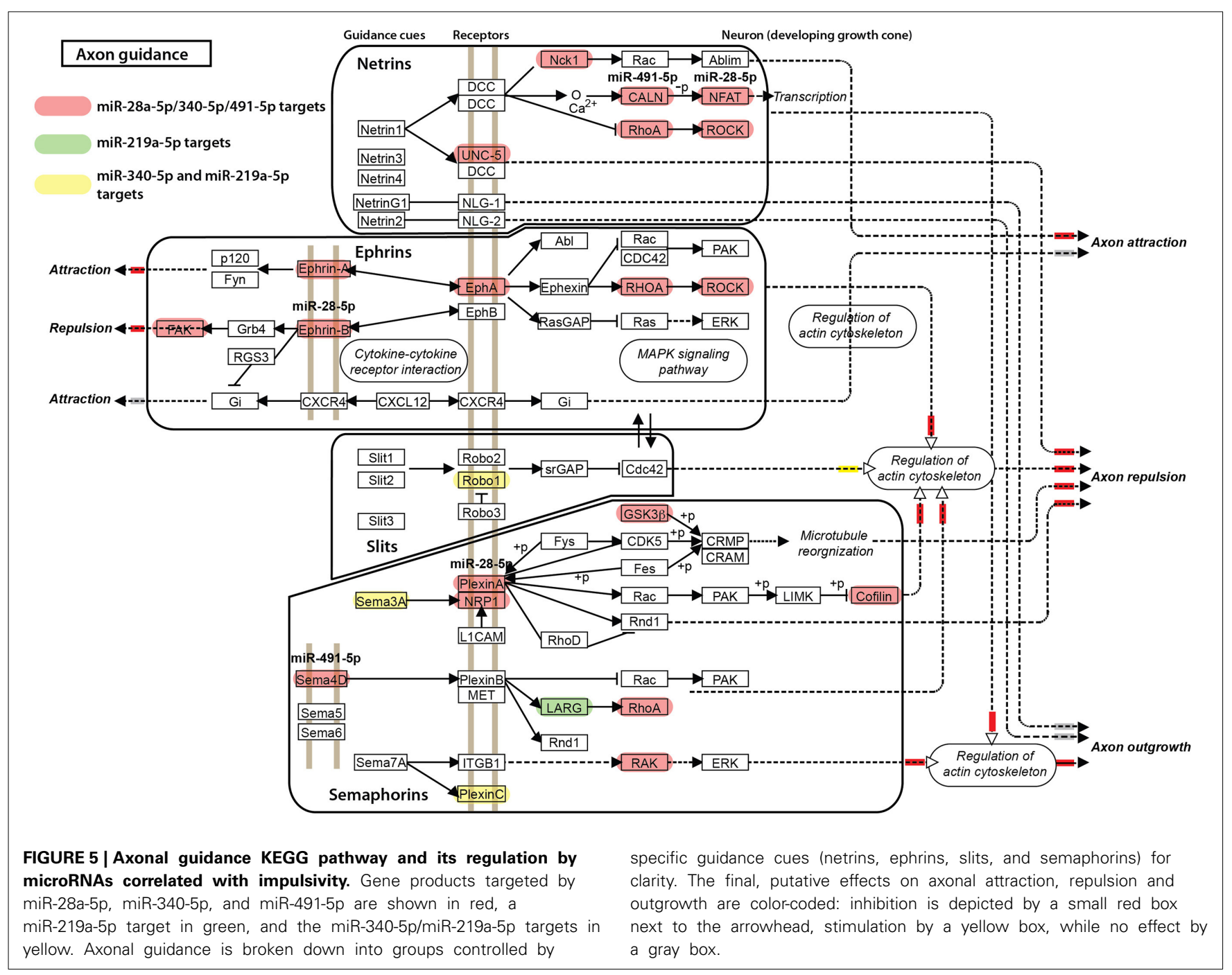

association occurs early in life, as impulsivity is a risk factor for child-obesity (van den Berg et al., 2011; Thamotharan et al., 2013) and obese adolescent individuals have been reported as being more impulsive (Delgado-Rico et al., 2013). This association persists into adulthood (Sutin et al., 2013). Moreover, in psychiatric patients with bipolar disorder the co-occurrence of impulsivity and obesity worsens the prognosis (Galvez et al., 2014).

Since their discovery about 10 years ago, miRNA regulation of gene expression became widely accepted as a fundamental biological process (Farh et al., 2005; Guo et al., 2010). Typically, miRNAs destabilize targeted mRNAs that subsequently undergo degradation. We showed here that expression of miR-190b-5p, but not other miRNAs targeting $\mathrm{Nrg} 3 \mathrm{mRNAs}$, is highly correlated with impulsivity. This correlation is negative, which supports a suppressive role of miR-190b-5p on Nrg3 gene expression. Moreover, expression of miR-190b-5p is negatively correlated with two body mass traits and food intake that significantly correlate with impulsivity (Figure 3). Based on this, we could hypothesize that the shared mechanism between impulsivity and compulsivity could lie in regulation of a miR-190b-5p-directed network.
One of the challenges in understanding miRNA mechanisms is the multiplicity of their actions, in which one miRNA can regulate several targets, and simultaneously a single target can be regulated by several miRNAs. Here, we focused on a selection of the network that is targeted by miR-190b-5p, and that is related to biological relevance of Nrg3. Nrg3 is a part of a complex, intertwined biological network, in which this growth factor can bind and activate Erbb4, a receptor tyrosine kinase (Zhang et al., 1997; Rochat et al., 2008). Erbb4 can upon activation regulate a wide range of biochemical pathways in a cell. Importantly, the expression of $\mathrm{Nrg} 3$ is mainly restricted to the nervous system (Zhang et al., 1997; Evenden, 1999). Mutations in Nrg3 have recently been reported to increase the risk for schizophrenia, and to alter activation of the PFC in humans (Kao et al., 2010; Gupta et al., 2011; Tost et al., 2014). In particular, Nrg3 and ErbB4 could play an important neurodevelopmental role contributing to aberrant cognitive function and social behaviors (Depue et al., 2014; Loos et al., 2014) (Koob, 2009; Koob and Volkow, 2010; Paterson and Law, 2014; Tost et al., 2014). However, Nrg3 has a role beyond this developmental period (Lee et al., 1993; Wightman et al., 
1993; Loos et al., 2014), probably maintaining synaptic structures during adulthood. In this respect, the role of miRNAs is important, because the network found for miR-190b-5p targets and associated genes includes those that have a function in synapse formation and stabilization. Recently, miR-190 has been shown to be involved in regulation of synapses by drugs of abuse. Fentanyl (but not morphine) decreases miR-190 levels and thereby regulates NeuroD by binding to its $3^{\prime}$-UTR (Ambros and Lee, 2004; Landgraf et al., 2007; Friedman et al., 2009; Zheng et al., 2010). NeuroD is critical for dendritic spine stability without affecting axon growth (Gaudillière et al., 2004; Siegel et al., 2011). One of the direct targets of the miR-190b-5p network is neuroligin (Nlgn1), which is a key synaptic factor that mediates the formation and maintenance of synapses. It acts as a cell adhesion protein expressed in the post-synaptic compartment, and interacts with $ß$-neurexins located pre-synaptically. Together, neuroligin and $B$ neurexins hold both synaptic compartments close to each other, helping to create a synaptic cleft. Importantly, alterations in expression of neuroligin are linked to psychiatric diseases, specifically autism spectrum disorders (Farh et al., 2005; Lewis et al., 2005; Südhof, 2008).

Besides miR-190b-5p, the expression of miR-340-5p, miR-28a and miR-491 in the amygdala was negatively correlated with impulsivity. Whereas miR-340-5p was detected as downregulated with age in serum (Im and Kenny, 2012; Xu et al., 2012; Nestler, 2014; Zhang et al., 2014), miR-340-5p was upregulated in the hippocampus of FMRP mice (Hébert and De Strooper, 2009; Liu et al., 2014), a neurodevelopmental model characterized by more immature dendritic spines (Bassell and Warren, 2008; Pietrzykowski, 2010). Likewise, in melanocytes, miR-340 is able to increase dendritic formation (Sánchez-Mora et al., 2013; Jian et al., 2014). In this respect, the negative correlation of miR-340$5 \mathrm{p}$ with impulsivity could relate to a decrease in synaptic plasticity in the amygdala of impulsive mice.

As yet, miR-28a-5p and miR-491-5p have been mainly studied in cancer, and more specifically in the formation of gliomas (Malzkorn et al., 2010; Németh et al., 2013; Li et al., 2014). Both derivatives of the mir-491 precursor, miR-491-5p and $-3 p$ control key hallmarks of glioma carcinogenesis, repressing proliferation (Li et al., 2014; Loos et al., 2014). In addition, in the PFC of depressed patients, as well as in serum of schizophrenic patients, a decrease in miR-491-3p is observed (Cole and Robbins, 1989; Shi et al., 2012; Serafini et al., 2014). Moreover, the expression of miR-219-5p, which was positively correlated with impulsivity, is downregulated in gliomas. This is in concordance with its role in inhibiting proliferation of medulloblastoma cells and migration of glioma cells, as well as promoting neural differentiation (Hudish et al., 2013; Rao et al., 2013; Shi et al., 2014). The role of miR-219 in the brain is limited to being downregulated upon disruption of NMDAR signaling in the PFC, and downregulation of miR-219 inhibits the NMDAR antagonist dizocilpine-induced effect on locomotion and stereotypy (Kocerha et al., 2009). Taken together, although a role for these miRNAs in alterating synaptic plasticity, in part, through the changes in development of spines or dendrites seems likely, this has not been established yet. Based on our results, we propose here that miR219-5p along with miR-340-5p, miR-28a-5p and possibly miR-491-5p could have a key role in the development of psychiatric diseases, possibly by affecting the balance between neuronal outgrowth and differentiation in the context of synapse plasticity, maturation and maintenance.

\section{CONCLUSIONS}

Our goal here was to use multiple bioinformatic resources to select miRNAs relevant to impulsivity. Our data suggest that miR190b-5p is a strong candidate of a biological network regulation centered around $\mathrm{Nrg} 3$ in relation to impulsive and compulsive traits. This network could affect synaptic processes in amygdala. On a critical note, these findings stem from big datasets, which also could have larger (technical) variation, and therefore need validation at multiple levels. On the other hand, the strict criteria that we have applied, in combination with the fact that we tested a selective hypothesis should be a more powerful approach reducing false-positives, as suggested before (Chesler et al., 2005), and therefore lead to a specific set of experiments that can be tested in the lab. These could entail performing quantitative gene expression (e.g., real-time PCR) for miRs, Nrg3 and target genes, as well as protein levels for $\mathrm{Nrg} 3$ and target genes (e.g., quantitative proteomics), in different BXD strains, or after intervention (e.g., RNA interference) of either levels of Nrg3 (Nrg3 KO, Nrg3 overexpression) or specific miRs. Although we focused here on the amygdala, because of its role in emotional aspects of impulsivity, it is evident that impulsivity stems from the complex interactions of several brain regions, including the striatum and cortical regions (Crews and Boettiger, 2009; Basar et al., 2010; Kerr et al., 2014). Therefore, it will be of great interest to show directly a role of these miRNAs in impulsivity and compulsivity in these brain regions by wet lab experiments, as mentioned above.

Psychiatric disorders are complex amalgamations of behavioral traits rooted in molecular mechanisms. With the emerging role of miRNAs as major regulators of gene and protein expression, and cellular function, they are also attractive targets for therapeutic approaches (Dinan, 2010; Chan and Kocerha, 2012)., We propose here that a set of miRNAs contributes to the regulation of synaptic plasticity in the amygdala, which could bring us a little bit closer to understanding mechanisms in psychiatric disorders and creating new therapeutic options.

\section{ACKNOWLEDGMENTS}

We would like to thank the organizers and participants of the first INCF Short Course on Neuroinformatics, Neurogenomics and Brain Disease (Fraueninsel, Bavaria, Germany), where the project was conceived, for their valuable contributions. This work was supported by NIH/NIAAA grants AA01748 and AA017920, as well as a Pilot Project from the NIH/NIAAA INIA-West consortium (AA020895) to Andrzej Z. Pietrzykowski, and by grants from the Center for Medical Systems Biology (CMSB) and HEALTH-2009-2.1.2-1 EU-FP7 "SynSys" (\#242167) to Sabine Spijker.

\section{REFERENCES}

Agnoli, L., and Carli, M. (2012). Dorsal-striatal 5-HT2A and 5-HT2C receptors control impulsivity and perseverative responding in the 5-choice serial reaction time task. Psychopharmacology (Berl.) 219, 633-645. doi: 10.1007/s00213-0112581-0 
Almasy, L., and Blangero, J. (2001). Endophenotypes as quantitative risk factors for psychiatric disease: rationale and study design. Am. J. Med. Genet. 105, 42-44. doi: 10.1002/1096-8628(20010108)105:13.3.CO;2-0

Ambros, V., and Lee, R. C. (2004). Identification of microRNAs and other tiny noncoding RNAs by cDNA cloning. Methods Mol. Biol. 265, 131-158. doi: 10.1385/ 1-59259-775-0:131

Arora, S., Rana, R., Chhabra, A., Jaiswal, A., and Rani, V. (2013). miRNAtranscription factor interactions: a combinatorial regulation of gene expression. Mol. Genet. Genomics 288, 77-87. doi: 10.1007/s00438-013-0734-Z

Bari, A., and Robbins, T. W. (2013). Inhibition and impulsivity: behavioral and neural basis of response control. Prog. Neurobiol. 108, 44-79. doi: 10.1016/j.pneurobio.2013.06.005

Barkley, R. A. (1997). Behavioral inhibition, sustained attention, and executive functions: constructing a unifying theory of ADHD. Psychol. Bull. 121, 65-94. doi: 10.1037/0033-2909.121.1.65

Barros, C. S., Calabrese, B., Chamero, P., Roberts, A. J., Korzus, E., Lloyd, K., et al. (2009). Impaired maturation of dendritic spines without disorganization of cortical cell layers in mice lacking NRG1/ErbB signaling in the central nervous system. Proc. Natl. Acad. Sci. USA 106, 4507-4512. doi: 10.1073/pnas.09003 55106

Basar, K., Sesia, T., Groenewegen, H., Steinbusch, H. W. M., Visser-Vandewalle, V., and Temel, Y. (2010). Nucleus accumbens and impulsivity. Prog. Neurobiol. 92, 533-557. doi: 10.1016/j.pneurobio.2010.08.007

Bassell, G. J., and Warren, S. T. (2008). Fragile X syndrome: loss of local mRNA regulation alters synaptic development and function. Neuron 60, 201-214. doi: 10.1016/j.neuron.2008.10.004

Bechara, A., and Van der Linden, M. (2005). Decision-making and impulse control after frontal lobe injuries. Curr. Opin. Neurol. 18, 734-739. doi: 10.1097/01.wco.0000194141.56429.3c

Bevilacqua, L., and Goldman, D. (2013). Genetics of impulsive behaviour. Philos. Trans. R. Soc. Lond. B Biol. Sci. 368:20120380. doi: 10.1098/rstb.2012. 0380

Callesen, M. B., Scheel-Krüger, J., Kringelbach, M. L., and Møller, A. (2013). A systematic review of impulse control disorders in Parkinson's disease. J. Parkinsons Dis. 3, 105-138. doi: 10.3233/JPD-120165

Chan, A. W. S., and Kocerha, J. (2012). The path to microRNA therapeutics in psychiatric and neurodegenerative disorders. Front. Genet. 3:82. doi: 10.3389/fgene. 2012.00082

Chesler, E. J., Lu, L., Shou, S., Qu, Y., Gu, J., Wang, J., et al. (2005). Complex trait analysis of gene expression uncovers polygenic and pleiotropic networks that modulate nervous system function. Nat. Genet. 37, 233-242. doi: $10.1038 / \mathrm{ng} 1518$

Cole, B. J., and Robbins, T. W. (1989). Effects of 6-hydroxydopamine lesions of the nucleus accumbens septi on performance of a 5-choice serial reaction time task in rats: implications for theories of selective attention and arousal. Behav. Brain Res. 33, 165-179. doi: 10.1016/S0166-4328(89)80048-8

Counotte, D. S., Goriounova, N. A., Li, K. W., Loos, M., van der Schors, R. C., Schetters, D., et al. (2011). Lasting synaptic changes underlie attention deficits caused by nicotine exposure during adolescence. Nat. Neurosci. 14, 417-419. doi: 10.1038/nn.2770

Crews, F. T., and Boettiger, C. A. (2009). Impulsivity, frontal lobes and risk for addiction. Pharmacol. Biochem. Behav. 93, 237-247. doi: 10.1016/j.pbb.2009.04.018

Crosbie, J., Arnold, P., Paterson, A., Swanson, J., Dupuis, A., Li, X., et al. (2013). Response inhibition and ADHD traits: correlates and heritability in a community sample. J. Abnorm. Child. Psychol. 41, 497-507. doi: 10.1007/s10802-0129693-9

de Geus, E. J., Wright, M. J., Martin, N. G., and Boomsma, D. I. (2001). Genetics of brain function and cognition. Behav. Genet. 31, 489-495. doi: 10.1023/A:1013360909048

Delgado-Rico, E., Soriano-Mas, C., Verdejo-Román, J., Río-Valle, J. S., and VerdejoGarcía, A. (2013). Decreased insular and increased midbrain activations during decision-making under risk in adolescents with excess weight. Obesity (Silver Spring) 21, 1662-1668. doi: 10.1002/oby.20375

Depue, B. E., Olson-Madden, J. H., Smolker, H. R., Rajamani, M., Brenner, L. A., and Banich, M. T. (2014). Reduced amygdala volume is associated with deficits in inhibitory control: a voxel- and surface-based morphometric analysis of comorbid PTSD/mild TBI. Biomed. Res. Int. 2014:691505. doi: $10.1155 / 2014 / 691505$
Dinan, T. G. (2010). MicroRNAs as a target for novel antipsychotics: a systematic review of an emerging field. Int. J. Neuropsychopharmacol. 13, 395-404. doi: 10.1017/S1461145709990800

Evenden, J. (1999). Impulsivity: a discussion of clinical and experimental findings. J. Psychopharmacol. (Oxford) 13, 180-192. doi: 10.1177/026988119901300211

Farh, K. K.-H., Grimson, A., Jan, C., Lewis, B. P., Johnston, W. K., Lim, L. P., et al. (2005). The widespread impact of mammalian MicroRNAs on mRNA repression and evolution. Science 310, 1817-1821. doi: 10.1126/science. 1121158

Friedman, R. C., Farh, K. K.-H., Burge, C. B., and Bartel, D. P. (2009). Most mammalian mRNAs are conserved targets of microRNAs. Genome Res. 19, 92-105. doi: 10.1101/gr.082701.108

Galvez, J. F., Bauer, I. E., Sanches, M., Wu, H. E., Hamilton, J. E., Mwangi, B., et al. (2014). Shared clinical associations between obesity and impulsivity in rapid cycling bipolar disorder: a systematic review. J. Affect. Disord. 168C, 306-313. doi: 10.1016/j.jad.2014.05.054

Gaudillière, B., Konishi, Y., de la Iglesia, N., Yao, G. L., and Bonni, A. (2004). A CaMKII-NeuroD signaling pathway specifies dendritic morphogenesis. Neuron 41, 229-241. doi: 10.1016/S0896-6273(03)00841-9

Görisch, J., and Schwarting, R. K. W. (2006). Wistar rats with high versus low rearing activity differ in radial maze performance. Neurobiol. Learn. Mem. 86, 175-187. doi: 10.1016/j.nlm.2006.02.004

Griffiths-Jones, S. (2006). miRBase: the microRNA sequence database. Methods Mol. Biol. 342, 129-138. doi: 10.1385/1-59745-123-1:129

Griffiths-Jones, S., Grocock, R. J., van Dongen, S., Bateman, A., and Enright, A. J. (2006). miRBase: microRNA sequences, targets and gene nomenclature. Nucleic Acids Res. 34, D140-D144. doi: 10.1093/nar/gkj112

Guo, H., Ingolia, N. T., Weissman, J. S., and Bartel, D. P. (2010). Mammalian microRNAs predominantly act to decrease target mRNA levels. Nature 466, 835-840. doi: 10.1038/nature09267

Gupta, R., Koscik, T. R., Bechara, A., and Tranel, D. (2011). The amygdala and decision-making. Neuropsychologia 49, 760-766. doi: 10.1016/j.neuropsychologia.2010.09.029

Hébert, S. S., and De Strooper, B. (2009). Alterations of the microRNA network cause neurodegenerative disease. Trends Neurosci. 32, 199-206. doi: 10.1016/j.tins.2008.12.003

Hochberg, Y., and Benjamini, Y. (1990). More powerful procedures for multiple significance testing. Stat. Med. 9, 811-818. doi: 10.1002/sim.4780090710

Huang, D. W., Sherman, B. T., Tan, Q., Collins, J. R., Alvord, W. G., Roayaei, J., et al. (2007). The DAVID Gene Functional Classification Tool: a novel biological module-centric algorithm to functionally analyze large gene lists. Genome Biol. 8:R183. doi: 10.1186/gb-2007-8-9-r183

Hudish, L. I., Blasky, A. J., and Appel, B. (2013). miR-219 regulates neural precursor differentiation by direct inhibition of apical par polarity proteins. Dev. Cell 27, 387-398. doi: 10.1016/j.devcel.2013.10.015

Humby, T., Wilkinson, L., and Dawson, G. (2005). Assaying aspects of attention and impulse control in mice using the 5-choice serial reaction time task. Curr. Protoc. Neurosci. Chapter 8:Unit 8.5H. doi: 10.1002/0471142301.ns08 $05 \mathrm{hs} 31$

Im, H.-I., and Kenny, P. J. (2012). MicroRNAs in neuronal function and dysfunction. Trends Neurosci. 35, 325-334. doi: 10.1016/j.tins.2012.01.004

Jian, Q., An, Q., Zhu, D., Hui, K., Liu, Y., Chi, S., et al. (2014). MicroRNA 340 Is involved in UVB-induced dendrite formation through the regulation of RhoA expression in melanocytes. Mol. Cell. Biol. 34, 3407-3420. doi: 10.1128/MCB.00106-14

Kanehisa, M., and Goto, S. (2000). KEGG: kyoto encyclopedia of genes and genomes. Nucleic Acids Res. 28, 27-30. doi: 10.1093/nar/28.1.27

Kao, W.-T., Wang, Y., Kleinman, J. E., Lipska, B. K., Hyde, T. M., Weinberger, D. R., et al. (2010). Common genetic variation in Neuregulin 3 (NRG3) influences risk for schizophrenia and impacts NRG3 expression in human brain. Proc. Natl. Acad. Sci. USA 107, 15619-15624. doi: 10.1073/pnas.1005410107

Kerr, K. L., Avery, J. A., Barcalow, J. C., Moseman, S. E., Bodurka, J., Bellgowan, P. S. F., et al. (2014). Trait impulsivity is related to ventral ACC and amygdala activity during primary reward anticipation. Soc. Cogn. Affect. Neurosci. doi: 10.1093/ scan/nsu023. [Epub ahead of print].

Kocerha, J., Faghihi, M. A., Lopez-Toledano, M. A., Huang, J., Ramsey, A. J., Caron, M. G., et al. (2009). MicroRNA-219 modulates NMDA receptor-mediated neurobehavioral dysfunction. Proc. Natl. Acad. Sci. USA 106, 3507-3512. doi: 10.1073/pnas.0805854106 
Koob, G. F. (2009). Dynamics of neuronal circuits in addiction: reward, antireward, and emotional memory. Pharmacopsychiatry 42(Suppl. 1), S32-S41. doi: 10.1055/s-0029-1216356

Koob, G. F., and Le Moal, M. (1997). Drug abuse: hedonic homeostatic dysregulation. Science 278, 52-58. doi: 10.1126/science.278.5335.52

Koob, G. F., and Volkow, N. D. (2010). Neurocircuitry of addiction. Neuropsychopharmacology 35, 217-238. doi: 10.1038/npp.2009.110

Krishnan, V., and Nestler, E. J. (2011). Animal models of depression: molecular perspectives. Curr. Top. Behav. Neurosci. 7, 121-147.doi: 10.1007/7854_2010_108

Kuntsi, J., McLoughlin, G., and Asherson, P. (2006). Attention deficit hyperactivity disorder. Neuromol. Med. 8, 461-484. doi: 10.1385/NMM:8:4:461

Landgraf, P., Rusu, M., Sheridan, R., Sewer, A., Iovino, N., Aravin, A., et al. (2007). A mammalian microRNA expression atlas based on small RNA library sequencing. Cell 129, 1401-1414. doi: 10.1016/j.cell.2007.04.040

Lee, R. C., Feinbaum, R. L., and Ambros, V. (1993). The C. elegans heterochronic gene lin-4 encodes small RNAs with antisense complementarity to lin-14. Cell 75, 843-854. doi: 10.1016/0092-8674(93)90529-Y

Lewis, B. P., Burge, C. B., and Bartel, D. P. (2005). Conserved seed pairing, often flanked by adenosines, indicates that thousands of human genes are microRNA targets. Cell 120, 15-20. doi: 10.1016/j.cell.2004.12.035

Li, X., Liu, Y., Granberg, K. J., Wang, Q., Moore, L. M., Ji, P., et al. (2014). Two mature products of MIR-491 coordinate to suppress key cancer hallmarks in glioblastoma. Oncogene. doi: 10.1038/onc.2014.98. [Epub ahead of print].

Liu, T., Wan, R.-P., Tang, L.-J., Liu, S.-J., Li, H.-J., Zhao, Q.-H., et al. (2014). A MicroRNA profile in Fmrl knockout mice reveals MicroRNA expression alterations with possible roles in fragile X syndrome. Mol. Neurobiol. doi: 10.1007/ s12035-014-8770-1

Lombardo, L. E., Bearden, C. E., Barrett, J., Brumbaugh, M. S., Pittman, B., Frangou, S., et al. (2012). Trait impulsivity as an endophenotype for bipolar I disorder. Bipolar Disord. 14, 565-570. doi: 10.1111/j.1399-5618.2012. 01035.x

Loos, M., Mueller, T., Gouwenberg, Y., Wijnands, R., van der Loo, R. J., Birchmeier, C., et al. (2014). Neuregulin-3 in the mouse medial prefrontal cortex regulates impulsive action. Biol. Psychiatry 76, 648-655. doi: 10.1016/j.biopsych.2014. 02.011

Loos, M., Staal, J., Pattij, T., Neuro-Bsik Mouse Phenomics, Consortium, Smit, A. B., and Spijker, S. (2012). Independent genetic loci for sensorimotor gating and attentional performance in BXD recombinant inbred strains. Genes Brain Behav. 11, 147-156. doi: 10.1111/j.1601-183X.2011.00754.x

Loos, M., van der Sluis, S., Bochdanovits, Z., van Zutphen, I. J., Pattij, T., Stiedl, O., et al. (2009). Activity and impulsive action are controlled by different genetic and environmental factors. Genes Brain Behav. 8, 817-828. doi: 10.1111/j.1601183X.2009.00528.x

Malzkorn, B., Wolter, M., Liesenberg, F., Grzendowski, M., Stühler, K., Meyer, H. E., et al. (2010). Identification and functional characterization of microRNAs involved in the malignant progression of gliomas. Brain Pathol. 20, 539-550. doi: 10.1111/j.1750-3639.2009.00328.x

Maragkakis, M., Alexiou, P., Papadopoulos, G. L., Reczko, M., Dalamagas, T., Giannopoulos, G., et al. (2009). Accurate microRNA target prediction correlates with protein repression levels. BMC Bioinformatics 10:295. doi: 10.1186/14712105-10-295

McCall, R. D. (2011). HPNS seizure risk: a role for the Golgi-associated retrograde protein complex? Undersea. Hyperb Med. 38, 3-9.

McElroy, S. L., Pope, H. G., Keck, P. E., Hudson, J. I., Phillips, K. A., and Strakowski, S. M. (1996). Are impulse-control disorders related to bipolar disorder? Compr. Psychiatry 37, 229-240. doi: 10.1016/S0010-440X(96)90001-2

Mulligan, M. K., Dubose, C., Yue, J., Miles, M. F., Lu, L., and Hamre, K. M. (2013). Expression, covariation, and genetic regulation of miRNA Biogenesis genes in brain supports their role in addiction, psychiatric disorders, and disease. Front. Genet. 4:126. doi: 10.3389/fgene.2013.00126

Murphy, C. M., Stojek, M. K., and Mackillop, J. (2013). Interrelationships among impulsive personality traits, food addiction, and body mass index. Appetite 73, 45-50. doi: 10.1016/j.appet.2013.10.008

Németh, N., Kovács-Nagy, R., Székely, A., Sasvári-Székely, M., and Rónai, Z. (2013). Association of impulsivity and polymorphic microRNA-641 target sites in the SNAP-25 gene. PLOS ONE 8:e84207. doi: 10.1371/journal.pone. 0084207

Nestler, E. J. (2014). Epigenetic mechanisms of drug addiction. Neuropharmacology 76(Pt B), 259-268. doi: 10.1016/j.neuropharm.2013.04.004
Nestor, P. G. (2002). Mental disorder and violence: personality dimensions and clinical features. Am. J. Psychiatry 159, 1973-1978. doi: 10.1176/appi.ajp.159.12.1973

Palanza, P. (2001). Animal models of anxiety and depression: how are females different? Neurosci. Biobehav. Rev. 25, 219-233. doi: 10.1016/S01497634(01)00010-0

Paterson, C., and Law, A. J. (2014). Transient overexposure of neuregulin 3 during early postnatal development impacts selective behaviors in adulthood. PLoS ONE 9:e104172. doi: 10.1371/journal.pone.0104172

Peirce, J. L., Lu, L., Gu, J., Silver, L. M., and Williams, R. W. (2004). A new set of BXD recombinant inbred lines from advanced intercross populations in mice. BMC Genet. 5:7. doi: 10.1186/1471-2156-5-7

Pietrzykowski, A. Z. (2010). The role of microRNAs in drug addiction: a big lesson from tiny molecules. Int. Rev. Neurobiol. 91, 1-24. doi: 10.1016/S00747742(10)91001-5

Plomin, R., McClearn, G. E., and Gora-Maslak, G. (1991). Quantitative trait loci and psychopharmacology. J. Psychopharmacol. (Oxford) 5, 1-9. doi: $10.1177 / 026988119100500102$

Puumala, T., Ruotsalainen, S., Jäkälä, P., Koivisto, E., Riekkinen, P., and Sirviö, J. (1996). Behavioral and pharmacological studies on the validation of a new animal model for attention deficit hyperactivity disorder. Neurobiol. Learn. Mem. 66, 198-211. doi: 10.1006/nlme.1996.0060

Rao, S. A. M., Arimappamagan, A., Pandey, P., Santosh, V., Hegde, A. S., Chandramouli, B. A., et al. (2013). miR-219-5p inhibits receptor tyrosine kinase pathway by targeting EGFR in glioblastoma. PLOS ONE 8:e63164. doi: 10.1371/journal.pone.0063164

Rebhan, M., Chalifa-Caspi, V., Prilusky, J., and Lancet, D. (1997). GeneCards: integrating information about genes, proteins and diseases. Trends Genet. 13, 163. doi: 10.1016/S0168-9525(97)01103-7

Robbins, T. W., Gillan, C. M., Smith, D. G., de Wit, S., and Ersche, K. D. (2012). Neurocognitive endophenotypes of impulsivity and compulsivity: towards dimensional psychiatry. Trends Cogn. Sci. (Regul. Ed.) 16, 81-91. doi: 10.1016/j.tics.2011.11.009

Rochat, L., Delbeuck, X., Billieux, J., d'Acremont, M., Van der Linden, A.C. J., and Van der Linden, M. (2008). Assessing impulsivity changes in Alzheimer disease. Alzheimer Dis. Assoc. Disord. 22, 278-283. doi: 10.1097/WAD.0b013e318166d692

Rosen, G. D., Chesler, E. J., Manly, K. F., and Williams, R. W. (2007). An informatics approach to systems neurogenetics. Methods Mol. Biol. 401, 287-303. doi: 10.1007/978-1-59745-520-6_16

Sachs, G. S., Baldassano, C. F., Truman, C. J., and Guille, C. (2000). Comorbidity of attention deficit hyperactivity disorder with early- and late-onset bipolar disorder. Am. J. Psychiatry 157, 466-468. doi: 10.1176/appi.ajp.157.3.466

Sánchez-Mora, C., Ramos-Quiroga, J.-A., Garcia-Martínez, I., Fernàndez-Castillo, N., Bosch, R., Richarte, V., et al. (2013). Evaluation of single nucleotide polymorphisms in the miR-183-96-182 cluster in adulthood attention-deficit and hyperactivity disorder (ADHD) and substance use disorders (SUDs). Eur. Neuropsychopharmacol. 23, 1463-1473. doi: 10.1016/j.euroneuro.2013. 07.002

Schachar, R. J., Forget-Dubois, N., Dionne, G., Boivin, M., and Robaey, P. (2011). Heritability of response inhibition in children. J. Int. Neuropsychol. Soc. 17, 238-247. doi: 10.1017/S1355617710001463

Serafini, G., Pompili, M., Hansen, K. F., Obrietan, K., Dwivedi, Y., Shomron, N., et al. (2014). The involvement of microRNAs in major depression, suicidal behavior, and related disorders: a focus on miR-185 and miR-491-3p. Cell. Mol. Neurobiol. 34, 17-30. doi: 10.1007/s10571-013-9997-5

Shi, J.-A., Lu, D.-L., Huang, X., and Tan, W. (2014). miR-219 inhibits the proliferation, migration and invasion of medulloblastoma cells by targeting CD164. Int. J. Mol. Med. 34, 237-243. doi: 10.3892/ijmm.2014.1749

Shi, W., Du, J., Qi, Y., Liang, G., Wang, T., Li, S., et al. (2012). Aberrant expression of serum miRNAs in schizophrenia. J. Psychiatr. Res. 46, 198-204. doi: 10.1016/j.jpsychires.2011.09.010

Siegel, G., Saba, R., and Schratt, G. (2011). microRNAs in neurons: manifold regulatory roles at the synapse. Curr. Opin. Genet. Dev. 21, 491-497. doi: 10.1016/j.gde.2011.04.008

Südhof, T. C. (2008). Neuroligins and neurexins link synaptic function to cognitive disease. Nature 455, 903-911. doi: 10.1038/nature07456

Sutin, A. R., Costa, P. T., Chan, W., Milaneschi, Y., Eaton, W. W., Zonderman, A. B., et al. (2013). I know not to, but i can't help it: weight gain and changes in 
impulsivity-related personality traits. Psychol. Sci. 24, 1323-1328. doi: 10.1177/ 0956797612469212

Thamotharan, S., Lange, K., Zale, E. L., Huffhines, L., and Fields, S. (2013). The role of impulsivity in pediatric obesity and weight status: a meta-analytic review. Clin. Psychol. Rev. 33, 253-262. doi: 10.1016/j.cpr.2012.12.001

Tost, H., Callicott, J. H., Rasetti, R., Vakkalanka, R., Mattay, V. S., Weinberger, D. R., et al. (2014). Effects of neuregulin 3 genotype on human prefrontal cortex physiology. J. Neurosci. 34, 1051-1056. doi: 10.1523/JNEUROSCI.3496-13.2014

van den Berg, L., Pieterse, K., Malik, J. A., Luman, M., Willems van Dijk, K., Oosterlaan, J., et al. (2011). Association between impulsivity, reward responsiveness and body mass index in children. Int. J. Obes. (Lond) 35, 1301-1307. doi: 10.1038/ijo.2011.116

Vlachos, I. S., Kostoulas, N., Vergoulis, T., Georgakilas, G., Reczko, M., Maragkakis, M., et al. (2012). DIANA miRPath v.2.0: investigating the combinatorial effect of microRNAs in pathways. Nucleic Acids Res. 40, W498-W504. doi: $10.1093 /$ nar/gks494

Warde-Farley, D., Donaldson, S. L., Comes, O., Zuberi, K., Badrawi, R., Chao, P., et al. (2010). The GeneMANIA prediction server: biological network integration for gene prioritization and predicting gene function. Nucleic Acids Res. 38, W214-W220. doi: 10.1093/nar/gkq537

Wightman, B., Ha, I., and Ruvkun, G. (1993). Posttranscriptional regulation of the heterochronic gene lin-14 by lin-4 mediates temporal pattern formation in C. elegans. Cell 75, 855-862. doi: 10.1016/0092-8674(93)90530-4

Williams, R. W., Gu, J., Qi, S., and Lu, L. (2001). The genetic structure of recombinant inbred mice: high-resolution consensus maps for complex trait analysis. Genome Biol. 2:RESEARCH0046. doi: 10.1186/gb-2001-2-8

Wolters, E. C., van der Werf, Y. D., and van den Heuvel, O. A. (2008). Parkinson's disease-related disorders in the impulsive-compulsive spectrum. J. Neurol. 255(Suppl. 5), 48-56. doi: 10.1007/s00415-008-5010-5

Xu, B., Hsu, P.-K., Karayiorgou, M., and Gogos, J. A. (2012). MicroRNA dysregulation in neuropsychiatric disorders and cognitive dysfunction. Neurobiol. Dis. 46, 291-301. doi: 10.1016/j.nbd.2012.02.016

Zhang, D., Sliwkowski, M. X., Mark, M., Frantz, G., Akita, R., Sun, Y., et al. (1997). Neuregulin-3 (NRG3): a novel neural tissue-enriched protein that binds and activates ErbB4. Proc. Natl. Acad. Sci. USA 94, 9562-9567. doi: 10.1073/pnas.94. 18.9562

Zhang, H., Yang, H., Zhang, C., Jing, Y., Wang, C., Liu, C., et al. (2014). Investigation of MicroRNA expression in human serum during the aging process. J. Gerontol. A Biol. Sci. Med. Sci. doi: 10.1093/gerona/glu145. [Epub ahead of print].

Zheng, H., Chu, J., Zeng, Y., Loh, H. H., and Law, P.-Y. (2010). Yin Yang 1 phosphorylation contributes to the differential effects of mu-opioid receptor agonists on microRNA-190 expression. J. Biol. Chem. 285, 21994-22002. doi: 10.1074/jbc. M110.112607

Zhu, S., Shi, R., Wang, J., Wang, J.-F., and Li, X.-M. (2014). Unpredictable chronic mild stress not chronic restraint stress induces depressive behaviours in mice. Neuroreport 25, 1151-1155. doi: 10.1097/WNR.0000000000000243

Conflict of Interest Statement: Sabine Spijker and Andrzej Z.Pietrzykowski received a partial restitution for the travel to the INCF Short Course on Neuroinformatics, Neurogenomics and Brain Disease. The authors declare that the research was conducted in the absence of any commercial or financial relationships that could be construed as a potential conflict of interest.

Received: 12 September 2014; accepted: 13 November 2014; published online: 11 December 2014.

Citation: Pietrzykowski AZ and Spijker S (2014) Impulsivity and comorbid traits: a multi-step approach for finding putative responsible microRNAs in the amygdala. Front. Neurosci. 8:389. doi: 10.3389/fnins.2014.00389

This article was submitted to Neurogenomics, a section of the journal Frontiers in Neuroscience.

Copyright (c) 2014 Pietrzykowski and Spijker. This is an open-access article distributed under the terms of the Creative Commons Attribution License (CC BY). The use, distribution or reproduction in other forums is permitted, provided the original author(s) or licensor are credited and that the original publication in this journal is cited, in accordance with accepted academic practice. No use, distribution or reproduction is permitted which does not comply with these terms. 